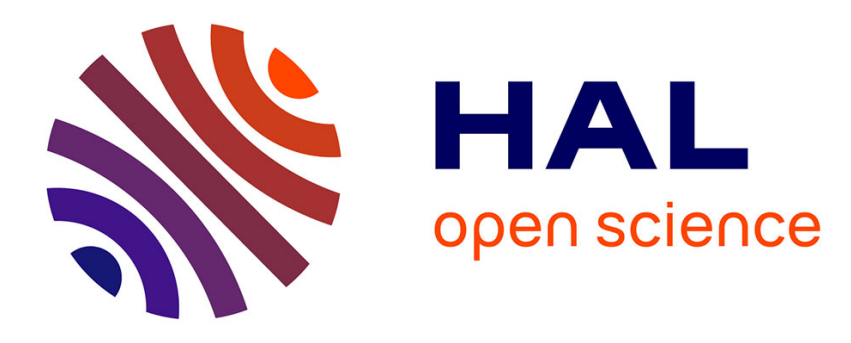

\title{
Riemann-Hilbert problem approach for two-dimensional flow inverse scattering
}

\author{
Alexey Agaltsov, Roman Novikov
}

\section{To cite this version:}

Alexey Agaltsov, Roman Novikov. Riemann-Hilbert problem approach for two-dimensional flow inverse scattering. Journal of Mathematical Physics, 2014, 55 (10), pp.103502. hal-00939283

\section{HAL Id: hal-00939283 https://hal.science/hal-00939283}

Submitted on 30 Jan 2014

HAL is a multi-disciplinary open access archive for the deposit and dissemination of scientific research documents, whether they are published or not. The documents may come from teaching and research institutions in France or abroad, or from public or private research centers.
L'archive ouverte pluridisciplinaire HAL, est destinée au dépôt et à la diffusion de documents scientifiques de niveau recherche, publiés ou non, émanant des établissements d'enseignement et de recherche français ou étrangers, des laboratoires publics ou privés. 


\title{
Riemann-Hilbert problem approach for two-dimensional flow inverse scattering*
}

\author{
A.D. Agaltsoथ, R. G. Novikov 2
}

\begin{abstract}
We consider inverse scattering for the time-harmonic wave equation with first-order perturbation in two dimensions. This problem arises in particular in the acoustic tomography of moving fluid. We consider linearized and nonlinearized reconstruction algorithms for this problem of inverse scattering. Our nonlinearized reconstruction algorithm is based on the non-local Riemann-Hilbert problem approach. Comparisons with preceding results are given.
\end{abstract}

Key words: acoustic tomography, moving fluid, wave equation with first-order perturbation, inverse scattering, Riemann-Hilbert problem.

\section{Introduction}

We consider the equation

$$
-\Delta \psi-2 i A(x) \nabla \psi+V(x) \psi=E \psi, \quad x=\left(x_{1}, x_{2}\right) \in \mathbb{R}^{2}, E>0,
$$

where $\Delta=\partial_{x_{1}}^{2}+\partial_{x_{2}}^{2}, \nabla=\left(\partial_{x_{1}}, \partial_{x_{2}}\right), \partial_{x_{k}}=\partial / \partial x_{k}, k=1,2$, and $A=\left(A_{1}, A_{2}\right)$ and $V$ are vector and scalar potentials on $\mathbb{R}^{2}$, respectively. In addition we assume that

$$
\begin{aligned}
& A_{1}, A_{2} \text { and } V \text { are sufficiently regular functions on } \mathbb{R}^{2} \\
& \text { with sufficient decay at infinity. }
\end{aligned}
$$

Equation (1.1) can be considered as a model equation for the time-harmonic $\exp (-i \omega t)$ acoustic pressure $\psi$ in a two-dimensional moving fluid, see e.g. [RW], [RE]. In this setting

$$
E=\left(\frac{\omega}{c_{0}}\right)^{2}, \quad A(x)=\frac{\omega}{c_{0}} u(x), \quad V(x)=\left(1-n^{2}(x)\right)\left(\frac{\omega}{c_{0}}\right)^{2},
$$

where $c_{0}$ is a reference sound speed, $n(x)$ is a scalar index of refraction, $u(x)$ is a normalized fluid velocity vector.

\footnotetext{
* The main part of the work was fulfilled during the stage of the first author in the Centre de Mathématiques Appliquées of Ecole Polytechnique in October-December 2013

${ }^{1}$ 119991, Lomonosov Moscow State University, Faculty of Computational Mathematics and Cybernetics, Moscow, Russia; email: agalets@gmail.com

${ }^{2}$ CNRS (UMR 7641), Centre de Mathématiques Appliquées, Ecole Polytechnique, 91128 Palaiseau, France; IEPT RAS, 117997, Moscow, Russia; Moscow Institute of Physics and Technology, Dolgoprudny, Russia; email: novikov@cmap.polytechnique.fr
} 
Equation (1.1) can be also considered as the two-dimensional Schrödinger equation at fixed energy $E$ with magnetic potential $A$ and electric potential $v$, where

$$
\begin{aligned}
V(x) & =A^{2}(x)-i \operatorname{div} A(x)+v(x) \\
& =A_{1}^{2}(x)+A_{2}^{2}(x)-i\left(\frac{\partial A_{1}(x)}{\partial x_{1}}+\frac{\partial A_{2}(x)}{\partial x_{2}}\right)+v(x),
\end{aligned}
$$

see e.g. [HN1], [ER2].

For equation (1.1) we consider the classical scattering solutions $\psi^{+}$continuous and bounded on $\mathbb{R}^{2}$ with their first derivatives and specified by the following asymptotics as $|x| \rightarrow \infty$ :

$$
\begin{aligned}
& \psi^{+}(x, k)=e^{i k x}+C(|k|) \frac{e^{i|k||x|}}{|x|^{1 / 2}} f\left(k,|k| \frac{x}{|x|}\right)+o\left(\frac{1}{|x|^{1 / 2}}\right), \\
& x \in \mathbb{R}^{2}, \quad k \in \mathbb{R}^{2}, k^{2}=E, \quad C(|k|)=-\pi i \sqrt{2 \pi} e^{-i \pi / 4}|k|^{1 / 2},
\end{aligned}
$$

where a priori unknown function $f=f(k, l), k, l \in \mathbb{R}^{2}, k^{2}=l^{2}=E$, arising in (1.5) is the classical scattering amplitude for (1.1).

Given potentials $A, V$, to determine $\psi^{+}$and $f$ one can use the following Lippmann-Schwinger integral equation (1.6) and formula (1.8) (see e.g. [HN1]):

$$
\begin{gathered}
\psi^{+}(x, k)=e^{i k x}+\int_{\mathbb{R}^{2}} G^{+}(x-y, k) \times \\
\times\left(-2 i A(y) \nabla_{y} \psi^{+}(y, k)+V(y) \psi^{+}(y, k)\right) d y, \\
G^{+}(x, k)=-(2 \pi)^{-2} \int_{\mathbb{R}^{2}} \frac{e^{i \xi x} d \xi}{\xi^{2}-k^{2}-i 0}=-\frac{i}{4} H_{0}^{1}(|x||k|),
\end{gathered}
$$

where $x \in \mathbb{R}^{2}, k \in \mathbb{R}^{2}, k^{2}=E, H_{0}^{1}$ is the Hankel function of the first kind;

$$
f(k, l)=(2 \pi)^{-2} \int_{\mathbb{R}^{2}} e^{-i l y}\left(-2 i A(y) \nabla_{y} \psi^{+}(y, k)+V(y) \psi^{+}(y, k)\right) d y,
$$

where $k \in \mathbb{R}^{2}, l \in \mathbb{R}^{2}, k^{2}=l^{2}=E$. Actually, we consider (1.6) and its differentiated version, where $\nabla$ is applied to both sided of (1.6), as a system of linear integral equations for $\psi^{+}$and $\nabla \psi^{+}$.

One can see that the scattering amplitude $f$ for equation (1.1) at fixed $E$ is defined on

$$
M_{E}=\left\{k \in \mathbb{R}^{2}, l \in \mathbb{R}^{2}: k^{2}=l^{2}=E\right\}, \quad E>0 .
$$

Note that $f$ on $M_{E}$ is invariant with respect to the gauge transformations

$$
\begin{aligned}
& A \rightarrow A+\nabla \varphi, \\
& V \rightarrow V-i \Delta \varphi+(\nabla \varphi)^{2}+2 A \nabla \varphi,
\end{aligned}
$$

where $\varphi$ is a sufficiently regular function on $\mathbb{R}^{2}$ with sufficient decay at infinity, see e.g. [HN2], [ER2]. In addition, $\psi^{+}$is transformed as

$$
\psi^{+} \rightarrow e^{-i \varphi} \psi^{+}
$$


with respect to 1.10 .

In this work we consider the following inverse scattering problem for equation (1.1) under assumptions (1.2):

Problem 1.1. Given scattering amplitude $f$ on $M_{E}$ at fixed $E>0$, find potentials $A$ and $V$ on $\mathbb{R}^{2}$ (at least approximately).

Problem 1.1 for the case when $A \equiv 0$ was studied, in particular, in [N1], [GM], [N3], [GN2], [N4], [BBMRS], [BAR] and in [N2], [Buk].

Problem 1.1 for the general case was considered, in particular, on page 457 of [N3] and in [X].

Problem 1.1 is also related with several other inverse problems for the Schrödinger equation in magnetic field (and for the time-harmonic wave equation with firstorder perturbation) in dimension $d \geq 2$. Concerning these other inverse problems see [DKN], [Sh], [HN2], [Nor], [RW], [RE], [BBS], [ER1], [NSU], [A], [ER2], [Ni], [WY], [GT], [IY] and references therein.

Note that approximate finding $A$ and $V$ in Problem 1.1 means, in particular, finding modulo transformations (1.10). However, for real-valued $A$ and $V$ there is no gauge nonuniqueness (1.10) in Problem 1.1]! In addition, $A$ and $V$ of formulas (1.3) (of moving fluid acoustics) are real if $n$ is real.

In this work we are mainly motivated by applications to the acoustic tomography of moving fluid discribed in [Nor], [RW], [RE], [BBS]. Note that in their reconstruction results works [Nor], [RW], [RE], [BBS] proceed from near-field scattering data (e.g. from some near field information on $\psi^{+}$) instead of the scattering amplitude $f$. But it is also known that near-field scattering data can be transformed into values of $f$, see e.g. [Ber], [BBS].

Results of the present work can be described as follows:

In Section 2 we give formulas for solving Problem 1.1 in the Born approximation. To our knowledge these formulas were not yet given explicitely in the literature for the case when $A \not \equiv 0$. These formulas are proved in Section 5 .

In Section 3 we give a nonlinearized reconstruction algorithm for Problem 1.1. For the case when $A \equiv 0$ this algorithm is reduced to the algorithm of [N4] and was implemented numerically in [BAR]. For the general case this algorithm can be also regarded as simplication and development of the algorithm mentioned on page 457 of [N3] and based on the Riemann-Hilbert-Manakov problem approach of [GN1], [N1]. A derivation of the reconstruction algorithm of Section 3 is given in Section 6.

In Section 4 we show that in the Born approximation the algorithm of Section 3 is reduced to formulas of Section 2. Related proofs are given in Section 7.

In a similar way with results of [NS] the reconstruction algorithm of Section 3 can be generalized to the multi-channel case. This generalization will be given in a subsequent work.

In the present work we are focused on approximate reconstructions for Problem 1.1, admitting stable numerical implementation. Issues related with theorems of uniqueness and examples of nonuniqueness for Problem 1.1 will be considered in a subsequent work. 


\section{Inverse scattering in the Born approximation}

If $A=\left(A_{1}, A_{2}\right)$ and $V$ satisfy (1.2) and are sufficiently small, then proceeding from (1.6), (1.8) we have the following Born approximation formulas for direct scattering:

$$
\begin{aligned}
& \psi^{+}(x, k) \approx e^{i k x}, \quad \nabla \psi^{+}(x, k) \approx e^{i k x} i k, \\
& f(k, l) \approx f^{\operatorname{lin}}(k, l), \\
& f^{\operatorname{lin}}(k, l) \stackrel{\text { def }}{=}(2 \pi)^{-2} \int_{\mathbb{R}^{2}} e^{i(k-l) x}(2 k A(x)+V(x)) d x,
\end{aligned}
$$

where $x, k, l \in \mathbb{R}^{2}, k^{2}=l^{2}=E$. In particular, formulas (2.1) can be specified as (2.14).

Note that $f^{\text {lin }}$ on $M_{E}$ is invariant with respect to the gauge transformations

$$
A \rightarrow A+\nabla \varphi, \quad V \rightarrow V-i \Delta \varphi
$$

where $\varphi$ is a sufficiently regular function on $\mathbb{R}^{2}$ with sufficient decay at infinity. This invariance follows from (2.2), (2.3), integrating by parts and using that $k^{2}-l^{2}=0$. We consider (2.3) as a linearization of (1.10) for small $A, V$ and $\varphi$.

In this section we consider the following linearized inverse scattering problem for equation (1.1) under assumptions (1.2):

Problem 2.1. Given linearized scattering amplitude $f^{\text {lin }}$ on $M_{E}$ at fixed $E>0$, find potentials $A$ and $V$ on $\mathbb{R}^{2}$ (at least approximately).

Note that approximate finding $A$ and $V$ in Problem 2.1 means, in particular, finding modulo transformations (2.3). However, in a similar way with Problem 1.1. there is no gauge nonuniqueness (2.3) in Problem 2.1 for the case of realvalued $A$ and $V$.

Problem 2.1 is a linearization of Problem 1.1 .

To study Problem 2.1 it is convenient to introduce $\varphi^{\text {div }}, A^{\text {div,0 }}, V^{\text {div,0 }}$ and $\varphi^{ \pm}, A^{ \pm, 0}, V^{ \pm, 0}$ such that:

$$
\begin{array}{r}
\Delta \varphi^{\operatorname{div}}(x)=-\operatorname{div} A(x), \quad \varphi^{\operatorname{div}}(x) \rightarrow 0 \text { as }|x| \rightarrow \infty, \\
A^{\operatorname{div}, 0}(x)=A(x)+\nabla \varphi^{\operatorname{div}}(x), \quad V^{\operatorname{div}, 0}(x)=V(x)-i \Delta \varphi^{\operatorname{div}}(x),
\end{array}
$$

where $x \in \mathbb{R}^{2}$;

$$
\begin{aligned}
\partial_{z} \varphi^{-}(x)=-\frac{1}{2}\left(A_{1}(x)-i A_{2}(x)\right), & \varphi^{-}(x) \rightarrow 0 \text { as }|x| \rightarrow \infty, \\
A^{-, 0}(x)=A(x)+\nabla \varphi^{-}(x), & V^{-, 0}(x)=V(x)-i \Delta \varphi^{-}(x), \\
\partial_{\bar{z}} \varphi^{+}(x)=-\frac{1}{2}\left(A_{1}(x)+i A_{2}(x)\right), & \varphi^{+}(x) \rightarrow 0 \text { as }|x| \rightarrow \infty, \\
A^{+, 0}(x)=A(x)+\nabla \varphi^{+}(x), & V^{+, 0}(x)=V(x)-i \Delta \varphi^{+}(x),
\end{aligned}
$$


where

$$
\partial_{z}=\frac{1}{2}\left(\partial_{x_{1}}-i \partial_{x_{2}}\right), \quad \partial_{\bar{z}}=\frac{1}{2}\left(\partial_{x_{1}}+i \partial_{x_{2}}\right), \quad x=\left(x_{1}, x_{2}\right) \in \mathbb{R}^{2} .
$$

One can see that

$$
\operatorname{div} A^{\operatorname{div}, 0}(x)=0, \quad A_{1}^{-, 0}(x)-i A_{2}^{-, 0}(x)=0, \quad A_{1}^{+, 0}(x)+i A_{2}^{+, 0}(x)=0,
$$

where $x \in \mathbb{R}^{2}, A^{ \pm, 0}=\left(A_{1}^{ \pm, 0}, A_{2}^{ \pm, 0}\right)$.

It is also convenient to transform formula (2.2) to the form

$$
\begin{aligned}
& f^{\operatorname{lin}}(k, l)-f^{\operatorname{lin}}(-l,-k)=2(k+l) \widehat{A}(k-l), \\
& f^{\operatorname{lin}}(k, l)+f^{\operatorname{lin}}(-l,-k)=2(k-l) \widehat{A}(k-l)+2 \widehat{V}(k-l), \\
& \widehat{A}(p)=(2 \pi)^{-2} \int_{\mathbb{R}^{2}} e^{i p x} A(x) d x, \quad \widehat{V}(p)=(2 \pi)^{-2} \int_{\mathbb{R}^{2}} e^{i p x} V(x) d x,
\end{aligned}
$$

where $(k, l) \in M_{E}, p \in \mathbb{R}^{2}$.

Note that

$$
\begin{aligned}
(k, l) \in M_{E} & \Longrightarrow k-l \in B_{2 \sqrt{E}}, \\
p \in B_{2 \sqrt{E}} & \Longrightarrow p=k-l \text { for some }(k, l) \in M_{E},
\end{aligned}
$$

where

$$
B_{r}=\left\{p \in \mathbb{R}^{2}:|p| \leq r\right\}, \quad r>0 .
$$

We define

$$
\begin{gathered}
C^{N, \sigma}\left(\mathbb{R}^{2}\right)=\left\{u \in C^{N}\left(\mathbb{R}^{2}\right):\|u\|_{N, \sigma}<+\infty\right\}, \\
\|u\|_{N, \sigma}=\max _{|n| \leqslant N} \sup _{x \in \mathbb{R}^{2}}\left(1+|x|^{2}\right)^{\sigma / 2}\left|\partial^{n} u(x)\right|, \quad N \in \mathbb{N} \cup\{0\}, \sigma \geqslant 0,
\end{gathered}
$$

where $C^{N}\left(\mathbb{R}^{2}\right)$ is the space of $N$-times continously differentiable complex-valued functions on $\mathbb{R}^{2}$,

$$
\partial^{n}=\partial_{x_{1}}^{n_{1}} \partial_{x_{2}}^{n_{2}}, \quad n=\left(n_{1}, n_{2}\right) \in(\mathbb{N} \cup\{0\})^{2}, \quad|n|=n_{1}+n_{2} .
$$

Note that if $A_{1}, A_{2}, V \in C^{0, \sigma}\left(\mathbb{R}^{2}\right)$ for some $\sigma>2$ and $\left\|A_{j}\right\|_{0, \sigma} \leq q, j=1,2$, $\|V\|_{0, \sigma} \leq q$, then

$$
\begin{gathered}
\psi^{+}(x, k)=e^{i k x}+O(q), \quad \nabla \psi^{+}(x, k)=e^{i k x} i k+O(q), \\
f(k, l)=f^{\operatorname{lin}}(k, l)+O\left(q^{2}\right) \quad \text { as } q \rightarrow+0,
\end{gathered}
$$

uniformly with repsect to $x, k, l \in \mathbb{R}^{2}, k^{2}=l^{2}=E$, at fixed $E>0$.

Theorem 2.1. Suppose that $A_{1}, A_{2}, V$ are real-valued and $A_{1}, A_{2}, V \in$ $C^{N, \sigma}\left(\mathbb{R}^{2}\right)$ for some $N \geq 3, \sigma>2$. Then the following formulas for solving 
Problem 2.1 are valid:

$$
\begin{gathered}
\widehat{A}(k-l)=\frac{f^{l i n}(k, l)-\overline{f^{l i n}(l, k)}}{2} \frac{k-l}{|k-l|^{2}}+\frac{f^{l i n}(k, l)-f^{l i n}(-l,-k)}{2} \frac{k+l}{|k+l|^{2}}, \\
\widehat{V}(k-l)=\frac{\overline{f^{l i n}(l, k)}+f^{\operatorname{lin}}(-l,-k)}{2},
\end{gathered}
$$

where $\widehat{A}, \widehat{V}$ are defined by (2.9) and $(k, l) \in M_{E}$;

$$
\begin{aligned}
A(x) & =A_{\text {appr }}(x, E)+A_{\text {err }}(x, E), \quad x \in \mathbb{R}^{2}, E>0, \\
A_{\text {appr }}(x, E) & =\int_{|p| \leq 2 \sqrt{E}} e^{-i p x} \widehat{A}(p) d p, \quad A_{\text {err }}(x, E)=\int_{|p| \geq 2 \sqrt{E}} e^{-i p x} \widehat{A}(p) d p, \\
V(x) & =V_{\text {appr }}(x, E)+V_{e r r}(x, E), \quad x \in \mathbb{R}^{2}, E>0, \\
V_{\text {appr }}(x, E) & =\int_{|p| \leq 2 \sqrt{E}} e^{-i p x} \widehat{V}(p) d p, \quad V_{\text {err }}(x, E)=\iint_{|p| \geq 2 \sqrt{E}} e^{-i p x} \widehat{V}(p) d p,
\end{aligned}
$$

where $\widehat{A}(p)$ and $\widehat{V}(p)$ for $|p| \leq 2 \sqrt{E}$ are given in terms of flin on $M_{E}$ according to (2.10), (2.15) and

$$
\begin{gathered}
\left|A_{e r r, j}(x, E)\right| \leq c_{1}(N, \sigma)\left\|A_{j}\right\|_{N, \sigma} E^{-\frac{N-2}{2}}, \\
\left|V_{e r r}(x, E)\right| \leq c_{1}(N, \sigma)\|V\|_{N, \sigma} E^{-\frac{N-2}{2}},
\end{gathered}
$$

where $x \in \mathbb{R}^{2}, j=1,2, A_{\text {err }}=\left(A_{\text {err }, 1}, A_{\text {err }, 2}\right), E \geq \frac{1}{4}$ and

$$
c_{1}(N, \sigma)=\frac{4}{(N-2)(\sigma-2)} \text {. }
$$

Theorem 2.2. Suppose that $A_{1}, A_{2}, V \in C^{N, \sigma}\left(\mathbb{R}^{2}\right)$ for some $N \geq 4$ and $\sigma>2$. Let $A^{\text {div, },}, V^{\text {div, }, 0}$ be defined according to (2.4). Then the following formulas for solving Problem 2.1 are valid:

$$
\begin{gathered}
\widehat{A}^{d i v, o}(k-l)=\frac{f^{l i n}(k, l)-f^{l i n}(-l,-k)}{2} \frac{k+l}{|k+l|^{2}}, \\
\widehat{V}^{d i v, o}(k-l)=\frac{f^{l i n}(k, l)+f^{l i n}(-l,-k)}{2},
\end{gathered}
$$

where $\widehat{A}^{d i v, 0}, \widehat{V}^{\text {div, }, 0}$ are the Fourier transforms of $A^{\text {div, },}, V^{\text {div, }, 0}$ (see (2.9)) and $(k, l) \in M_{E} ;$

$$
\begin{aligned}
& A^{d i v, O}(x)=A_{\text {appr }}^{d i v, O}(x, E)+A_{\text {err }}^{d i v, O}(x, E), \quad x \in \mathbb{R}^{2}, E>0, \\
& A_{\text {appr }}^{d i v, 0}(x, E)=\int_{|p| \leq 2 \sqrt{E}} e^{-i p x} \widehat{A}^{d i v, o}(p) d p, \quad A_{\text {err }}^{d i v, 0}(x, E)=\int_{|p| \geq 2 \sqrt{E}} e^{-i p x} \widehat{A}^{d i v, 0}(p) d p, \\
& V^{d i v, O}(x)=V_{\text {appr }}^{\text {div, }}(x, E)+V_{\text {err }}^{\text {div, }}(x, E), \quad x \in \mathbb{R}^{2}, E>0, \\
& V_{a p p r}^{d i v, o}(x, E)=\int_{|p| \leq 2 \sqrt{E}} e^{-i p x} \widehat{V}^{d i v, o}(p) d p, \quad V_{e r r}^{d i v, o}(x, E)=\iint_{|p| \geq 2 \sqrt{E}} e^{-i p x} \widehat{V}^{d i v, o}(p) d p,
\end{aligned}
$$


where $\widehat{A}^{d i v, O}(p)$ and $\widehat{V}^{d i v, 0}(p)$ for $|p| \leq 2 \sqrt{E}$ are given in terms of flin on $M_{E}$ according to (2.10), (2.21) and

$$
\begin{gathered}
\left|A_{e r r, j}^{d i v, o}(x, E)\right| \leq(1+\sqrt{2}) c_{1}(N, \sigma)\|A\|_{N, \sigma} E^{-\frac{N-2}{2}} \\
\left|V_{e r r}^{d i v, o}(x, E)\right| \leq c_{1}(N, \sigma)\left(\|V\|_{N, \sigma} E^{-\frac{N-2}{2}}+\sqrt{2}\|A\|_{N, \sigma} E^{-\frac{N-3}{2}}\right), \\
\|A\|_{N, \sigma}=\max \left(\left\|A_{1}\right\|_{N, \sigma},\left\|A_{2}\right\|_{N, \sigma}\right),
\end{gathered}
$$

where $x \in \mathbb{R}^{2}, j=1,2, E \geq \frac{1}{4}, A_{\text {err }}^{\text {div, } 0}=\left(A_{\text {err }, 1}^{\text {div }, 0}, A_{\text {err, } 2}^{\text {div, }}\right)$ and $c_{1}(N, \sigma)$ is defined by (2.20). Furthermore, if $\operatorname{div} A=0$ then $A^{\text {div }, O}=A, V^{\text {div, }, 0}=V$.

Theorem 2.3. Suppose that $A_{1}, A_{2}, V \in C^{N, \sigma}\left(\mathbb{R}^{2}\right)$ for some $N \geq 4$ and $\sigma>2$. Let $A^{ \pm, 0}, V^{ \pm, 0}$ be defined according to (2.5) -(2.6). Then the following formulas for solving Problem 2.1 are valid:

$$
\begin{gathered}
\widehat{A}_{1}^{ \pm, 0}(k-l)=\frac{1}{2} \frac{f(k, l)-f(-l,-k)}{k_{1}+l_{1} \pm i\left(k_{2}+l_{2}\right)}, \quad \widehat{A}_{2}^{ \pm, 0}(k-l)= \pm i \widehat{A}_{1}^{ \pm, 0}(k-l), \\
\widehat{V}^{ \pm, 0}(k-l)=\frac{\left(l_{1} \pm i l_{2}\right) f(k, l)+\left(k_{1} \pm i k_{2}\right) f(-l,-k)}{k_{1}+l_{1} \pm i\left(k_{2}+l_{2}\right)},
\end{gathered}
$$

where $\widehat{A}^{ \pm, 0}, \widehat{V}^{ \pm, 0}$ are the Fourier transforms of $A^{ \pm, 0}, V^{ \pm, 0}$ (see (2.9)) and $(k, l) \in M_{E}$

$$
\begin{gathered}
A^{ \pm, 0}(x)=A_{\text {appr }}^{ \pm, 0}(x, E)+A_{\text {err }}^{ \pm, 0}(x, E), \quad x \in \mathbb{R}^{2}, E>0 \\
A_{\text {appr }}^{ \pm, 0}(x, E)=\int_{|p| \leq 2 \sqrt{E}} e^{-i p x} \widehat{A}^{ \pm, 0}(p) d p, \quad A_{\text {err }}^{ \pm, 0}(x, E)=\iint_{|p| \geq 2 \sqrt{E}} e^{-i p x} \widehat{A}^{ \pm, 0}(p) d p, \\
V^{ \pm, 0}(x)=V_{\text {appr }}^{ \pm, 0}(x, E)+V_{\text {err }}^{ \pm, 0}(x, E), \quad x \in \mathbb{R}^{2}, E>0, \\
V_{\text {appr }}^{ \pm, 0}(x, E)=\int_{|p| \leq 2 \sqrt{E}} e^{-i p x} \widehat{V}^{ \pm, 0}(p) d p, \quad V_{\text {err }}^{ \pm, 0}(x, E)=\iint_{|p| \geq 2 \sqrt{E}} e^{-i p x} \widehat{V}^{ \pm, 0}(p) d p,
\end{gathered}
$$

where $\widehat{A}^{ \pm, 0}(p)$ and $\widehat{V}^{ \pm, 0}(p)$ for $|p| \leq 2 \sqrt{E}$ are given in terms of flin on $M_{E}$ according to (2.10), (2.27) and

$$
\begin{array}{r}
\left|A_{e r r, j}^{ \pm, 0}(x, E)\right| \leq(1+\sqrt{2}) c_{1}(N, \sigma)\|A\|_{N, \sigma} E^{-\frac{N-2}{2}}, \\
\left|V_{\text {err }}^{ \pm, 0}\right| \leq c_{1}(N, \sigma)\left(\|V\|_{N, \sigma} E^{-\frac{N-2}{2}}+\sqrt{2}\|A\|_{N, \sigma} E^{-\frac{N-3}{2}}\right),
\end{array}
$$

where $x \in \mathbb{R}^{2}, j=1,2, A_{\text {err }}^{ \pm, 0}=\left(A_{\text {err, }, 1}^{ \pm, 0}, A_{\text {err, }, \pm}^{ \pm, 0}\right),\|A\|_{N, \sigma}$ is defined by (2.23) and $c_{1}(N, \sigma)$ is given by (2.20). Furthermore, if $A_{1} \pm i A_{2}=0$ then $A=A^{ \pm, 0}$, $V=V^{ \pm, 0}$.

Theorems 2.1-2.3 are proved in Section 5 . 


\section{$3 \quad$ Nonlinearized inverse scattering}

3.1. Some notations. To study Problem 1.1 it is convenient to introduce $\varphi^{\text {div }}$, $A^{\text {div }}, V^{\text {div }}$ and $\varphi^{ \pm}, A^{ \pm}, V^{ \pm}$, where $\varphi^{\text {div }}$ and $\varphi^{ \pm}$are defined according to (2.4)(2.6) and

$$
\begin{gathered}
A^{\mathrm{div}}=A+\nabla \varphi^{\mathrm{div}}, \quad V^{\mathrm{div}}=V-i \Delta \varphi^{\mathrm{div}}+\left(\nabla \varphi^{\mathrm{div}}\right)^{2}+2 A \nabla \varphi^{\mathrm{div}}, \\
A^{ \pm}=A+\nabla \varphi^{ \pm}, \quad V^{ \pm}=V-i \Delta \varphi^{ \pm}+\left(\nabla \varphi^{ \pm}\right)^{2}+2 A \nabla \varphi^{ \pm} .
\end{gathered}
$$

In this section we give a nonlinearized algorithm for approximate finding $A^{ \pm}$, $V^{ \pm}$and $A^{\text {div }}, V^{\text {div }}$ on $\mathbb{R}^{2}$ from $f$ on $M_{E}$. This algorithm takes into account multiple scattering effects and can be regarded as a nonlinear version of formulas for $A_{\mathrm{appr}}^{ \pm, 0}, V_{\mathrm{appr}}^{ \pm, 0}, A_{\mathrm{appr}}^{\mathrm{div}, 0}, V_{\mathrm{appr}}^{\mathrm{div}, 0}$ of (2.28), (2.29), (2.22), (2.23).

It is convenient to use the following notations:

$$
\begin{gathered}
z=x_{1}+i x_{2}, \quad \bar{z}=x_{1}-i x_{2}, \\
\lambda=E^{-1 / 2}\left(k_{1}+i k_{2}\right), \quad \lambda^{\prime}=E^{-1 / 2}\left(l_{1}+i l_{2}\right),
\end{gathered}
$$

where $x=\left(x_{1}, x_{2}\right) \in \mathbb{R}^{2}, k=\left(k_{1}, k_{2}\right) \in \Sigma_{E}, l=\left(l_{1}, l_{2}\right) \in \Sigma_{E}$,

$$
\Sigma_{E}=\left\{m=\left(m_{1}, m_{2}\right) \in \mathbb{C}^{2}: m_{1}^{2}+m_{2}^{2}=E\right\}, \quad E>0 .
$$

In these notations

$$
\begin{gathered}
k_{1}=\frac{1}{2} E^{1 / 2}\left(\lambda+\lambda^{-1}\right), \quad k_{2}=\frac{i}{2} E^{1 / 2}\left(\lambda^{-1}-\lambda\right), \\
l_{1}=\frac{1}{2} E^{1 / 2}\left(\lambda^{\prime}+\lambda^{\prime-1}\right), \quad l_{2}=\frac{i}{2} E^{1 / 2}\left(\lambda^{\prime-1}-\lambda^{\prime}\right), \\
\exp (i k x)=\exp \left(\frac{i}{2} E^{1 / 2}\left(\lambda \bar{z}+\lambda^{-1} z\right)\right)
\end{gathered}
$$

where $\lambda, \lambda^{\prime} \in \mathbb{C} \backslash\{0\}, z \in \mathbb{C}^{2}, k, l \in \Sigma_{E}$.

In addition, using formulas (1.9), (3.3), (3.4), (3.5), (3.6) one can see that

$$
\begin{gathered}
\Sigma_{E} \cong \mathbb{C} \backslash\{0\}, \\
\Sigma_{E} \cap \mathbb{R}^{2}=\mathbb{S}_{\sqrt{E}}^{1} \cong T, \\
M_{E} \cong T \times T,
\end{gathered}
$$

where

$$
\begin{gathered}
\mathbb{S}_{r}^{1}=\left\{m \in \mathbb{R}^{2}:|m|=r\right\}, \quad r>0, \\
T=\{\lambda \in \mathbb{C}:|\lambda|=1\} .
\end{gathered}
$$

In addition, the functions $\psi^{+}, f$ of $(1.5)-(1.8)$ can be written as

$$
\psi^{+}=\psi^{+}(z, \lambda, E), \quad f=f\left(\lambda, \lambda^{\prime}, E\right)
$$

where $\lambda, \lambda^{\prime} \in T, z \in \mathbb{C}, E>0$. 
3.2. Reconstruction algorithm. Our nonlinearized algorithm for approximate finding $A^{ \pm}, V^{ \pm}$and $A^{\text {div }}, V^{\text {div }}$ on $\mathbb{R}^{2}$ from $f$ on $M_{E}$ has the following scheme

$$
f \longrightarrow h_{ \pm} \longrightarrow \mu^{+} \longrightarrow \mu_{ \pm} \longrightarrow A_{\mathrm{appr}}^{ \pm}, V_{\mathrm{appr}}^{ \pm} \longrightarrow A_{\mathrm{appr}}^{\mathrm{div}}, V_{\mathrm{appr}}^{\mathrm{div}}
$$

and consists of the following steps:

Step 1. Find functions $h_{ \pm}\left(\lambda, \lambda^{\prime}, E\right), \lambda, \lambda^{\prime} \in T$, from the following linear integral equations:

$$
\begin{gathered}
h_{ \pm}\left(\lambda, \lambda^{\prime}, E\right)-\pi i \int_{T} h_{ \pm}\left(\lambda, \lambda^{\prime \prime}, E\right) \chi\left( \pm i\left[\frac{\lambda}{\lambda^{\prime \prime}}-\frac{\lambda^{\prime \prime}}{\lambda}\right]\right) \times \\
\times f\left(\lambda^{\prime \prime}, \lambda^{\prime}, E\right)\left|d \lambda^{\prime \prime}\right|=f\left(\lambda, \lambda^{\prime}, E\right),
\end{gathered}
$$

where

$$
\chi(s)= \begin{cases}1 & \text { for } s \geq 0 \\ 0 & \text { for } s<0 .\end{cases}
$$

Step 2. Solve the following linear integral equation for $\mu^{+}(z, \lambda, E), z \in \mathbb{C}, \lambda \in T$, $E>0$ :

$$
\mu^{+}(z, \lambda, E)+\int_{T} B\left(\lambda, \lambda^{\prime}, z, E\right) \mu^{+}\left(z, \lambda^{\prime}, E\right)\left|d \lambda^{\prime}\right|=1,
$$

where

$$
\begin{gathered}
B\left(\lambda, \lambda^{\prime}, z, E\right)=\frac{1}{2} \int_{T} h_{-}\left(\zeta, \lambda^{\prime}, z, E\right) \chi\left(-i\left[\frac{\zeta}{\lambda^{\prime}}-\frac{\lambda^{\prime}}{\zeta}\right]\right) \frac{d \zeta}{\zeta-\lambda(1-0)}- \\
-\frac{1}{2} \int_{T} h_{+}\left(\zeta, \lambda^{\prime}, z, E\right) \chi\left(i\left[\frac{\zeta}{\lambda^{\prime}}-\frac{\lambda^{\prime}}{\zeta}\right]\right) \frac{d \zeta}{\zeta-\lambda(1+0)} \\
\quad h_{ \pm}\left(\lambda, \lambda^{\prime}, z, E\right) \stackrel{\text { def }}{=} h_{ \pm}\left(\lambda, \lambda^{\prime}, E\right) \times \\
\times \exp \left(-i \frac{\sqrt{E}}{2}\left(\left(\lambda-\lambda^{\prime}\right) \bar{z}+\left(\lambda^{-1}-\lambda^{\prime-1}\right) z\right)\right)
\end{gathered}
$$

and $\lambda, \lambda^{\prime} \in T, z \in \mathbb{C}, E>0$.

Step 3. Define functions $\mu_{ \pm}(z, \lambda, E), z \in \mathbb{C}, \lambda \in T, E>0$, by formulas

$$
\begin{gathered}
\mu_{ \pm}(z, \lambda, E)=\mu^{+}(z, \lambda, E)+\pi i \int_{T} h_{ \pm}\left(\lambda, \lambda^{\prime}, z, E\right) \times \\
\times \chi\left( \pm i\left[\frac{\lambda}{\lambda^{\prime}}-\frac{\lambda^{\prime}}{\lambda}\right]\right) \mu^{+}\left(z, \lambda^{\prime}, E\right)\left|d \lambda^{\prime}\right|
\end{gathered}
$$

where functions $h_{ \pm}\left(\lambda, \lambda^{\prime}, z, E\right)$ are given by (3.16) and $\chi$ is defined by $(3.13)$ 
Step 4. Functions $A_{\text {appr,j }}^{ \pm}(x, E), V_{\text {appr }}^{ \pm}(x, E), x \in \mathbb{R}^{2}, j=1,2, E>0$, are defined by formulas

$$
\begin{gathered}
A_{\mathrm{appr}, 1}^{-}(x, E)=\frac{i}{4} a_{z}^{-}(z, E), \quad A_{\mathrm{appr}, 2}^{-}(x, E)=\frac{1}{4} a_{z}^{-}(z, E), \\
a_{z}^{-}(z, E)=4 \partial_{\bar{z}} \ln \int_{T} \mu_{+}(z, \zeta, E)|d \zeta|, \\
V_{\mathrm{appr}}^{-}(x, E)=\frac{\sqrt{E}}{\pi} \int_{T} \partial_{z} \mu_{-}(z, \zeta, E) d \zeta,
\end{gathered}
$$

and

$$
\begin{gathered}
A_{\mathrm{appr}, 1}^{+}(x, E)=\frac{i}{4} a_{\bar{z}}^{+}(z, E), \quad A_{\mathrm{appr}, 2}^{+}(x, E)=-\frac{1}{4} a_{\bar{z}}^{+}(z, E), \\
a_{\bar{z}}^{+}(z, E)=-4 \partial_{z} \ln \int_{T} \mu_{+}(z, \zeta, E)|d \zeta|, \\
V_{\mathrm{appr}}^{+}(z, E)=2 i \sqrt{E} \partial_{\bar{z}}\left(\int_{T} \mu_{+}(z, \zeta, E) \frac{d \zeta}{\zeta^{2}} / \int_{T} \mu_{+}(z, \zeta, E) \frac{d \zeta}{\zeta}\right),
\end{gathered}
$$

where $z$ is given by (3.2) .

Step 5. Find $A_{\mathrm{appr}, \mathrm{j}}^{\mathrm{div}}(x, E), V_{\mathrm{appr}}^{\mathrm{div}}(x, E), x \in \mathbb{R}^{2}, j=1,2, E>0$, from formulas

$$
\begin{gathered}
A_{\mathrm{appr}, 1}^{\mathrm{div}}(x, E)=\frac{i}{8}\left(a_{z}^{-}(z, E)+a_{\bar{z}}^{+}(z, E)\right), \\
A_{\mathrm{appr}, 2}^{\mathrm{div}}(x, E)=\frac{1}{8}\left(a_{z}^{-}(z, E)-a_{\bar{z}}^{+}(z, E)\right), \\
V_{\mathrm{appr}}^{\mathrm{div}}(x, E)=\frac{1}{2}\left(V_{\mathrm{appr}}^{-}(x, E)+V_{\mathrm{appr}}^{+}(z, E)\right)-\frac{1}{8} a_{z}^{-}(z, E) a_{\bar{z}}^{+}(z, E),
\end{gathered}
$$

where $z$ is defined by (3.2) and functions $a_{z}^{-}, a_{\bar{z}}^{+}, V_{\text {appr }}^{ \pm}$are defined in (3.18), (3.19).

A derivation of this reconstruction algorithm is based on the method of the Riemann-Hilbert problem and on the $\bar{\partial}$-method. This derivation is given in Section 6 .

For the case when $A \equiv 0$ this algorithm is reduced to the algorithm of [N4] for approximatie finding $V$ on $\mathbb{R}^{2}$ from $f$ on $M_{E}$. The algorithm of [N4] consists of the same aforementioned steps 1,2,3 and the formula $V_{\text {appr }}=V_{\text {appr }}^{-}$, where $V_{\text {appr }}^{-}$is defined in (3.18). This algorithm of [N4] was implemented numerically in [BAR].

For the general case this algorithm can be also regarded as simplication and development of the algorithm mentioned (in few lines) on page 457 of [N3]. Actually, in [N3] the part of the algorithm consisting in finding $\mu_{ \pm}$from $h_{ \pm}$ 
is realized in a more complicated way. In addition, in [N3] the algorithm is mentioned for the case when

$$
A_{1}=\bar{A}_{1}, \quad A_{2}=\bar{A}_{2}, \quad-2 i \operatorname{div} \bar{A}+\bar{V}=V,
$$

i.e. for the self-adjoint case, whereas this assumption is not necessary for the algorithm.

3.3. Properties of the algorithm. Let

$$
\begin{gathered}
\left\|u_{1}\right\|_{L^{2}(T)}=\left(\int_{T}\left|u_{1}(\lambda)\right|^{2}|d \lambda|\right)^{1 / 2}, \\
\left\|u_{2}\right\|_{L^{2}\left(T^{2}\right)}=\left(\int_{T^{2}}\left|u_{2}\left(\lambda, \lambda^{\prime}\right)\right|^{2}|d \lambda|\left|d \lambda^{\prime}\right|\right)^{1 / 2}, \quad T^{2}=T \times T,
\end{gathered}
$$

where $u_{1}$ and $u_{2}$ are test functions on $T$ and $T^{2}$, respectively.

Proposition 3.1. Let $E>0$ be fixed. Suppose that

$$
f \in L^{2}\left(T^{2}\right), \quad\|f\|_{L^{2}\left(T^{2}\right)}<\frac{1}{\pi},
$$

where $f=f\left(\lambda, \lambda^{\prime}, E\right)$. Then equation (3.12) is uniquely solvable for $h_{ \pm} \in L^{2}\left(T^{2}\right)$ and

$$
\begin{aligned}
\left\|h_{ \pm}\right\|_{L^{2}\left(T^{2}\right)} & <\frac{\|f\|_{L^{2}\left(T^{2}\right)}}{1-\pi\|f\|_{L^{2}\left(T^{2}\right)}}, \\
\|B\|_{L^{2}\left(T^{2}\right)} & <\frac{2 \pi\|f\|_{L^{2}\left(T^{2}\right)}}{1-\pi\|f\|_{L^{2}\left(T^{2}\right)}},
\end{aligned}
$$

where $B$ is defined by (3.15), (3.16) (at fixed $z, E$ ). In addition, if

$$
\|f\|_{L^{2}\left(T^{2}\right)}<\frac{1}{3 \pi},
$$

then $\|B\|_{L^{2}\left(T^{2}\right)}<1$, equation (3.14), at fixed $z, E$, is uniquely solvable for $\mu^{+} \in L^{2}(T)$ and

$$
\begin{gathered}
\left\|\mu^{+}\right\|_{L^{2}(T)}<\frac{(2 \pi)^{1 / 2}}{1-\|B\|_{L^{2}\left(T^{2}\right)}}, \quad\left\|\mu^{+}-1\right\|_{L^{2}(T)}<\frac{(2 \pi)^{1 / 2}\|B\|_{L^{2}\left(T^{2}\right)}}{1-\|B\|_{L^{2}\left(T^{2}\right)}}, \\
\left\|\mu_{ \pm}-1\right\|_{L^{2}(T)}<\frac{3 \pi(2 \pi)^{1 / 2}\|f\|_{L^{2}\left(T^{2}\right)}}{1-3 \pi\|f\|_{L^{2}\left(T^{2}\right)}}
\end{gathered}
$$

where $\mu_{ \pm}$are defined by (3.17). In addition, at least, if

$$
\|f\|_{L^{2}\left(T^{2}\right)}<\frac{1}{6 \pi},
$$


then

$$
\int_{T} \mu_{+}(z, \lambda, E)|d \lambda| \neq 0 \quad \text { for all } z \in \mathbb{C},
$$

and $A_{a p p r, j}^{ \pm}, A_{a p p r, j}^{d i v}, j=1,2$, as well as $V_{\text {appr }}^{ \pm}, V_{\text {appr }}^{\text {div }}$ are bounded on $\mathbb{R}^{2}$.

Proposition 3.1 is based on solving the linear integral equations (3.12) and (3.14) by the method of successive approximations in $L^{2}\left(T^{2}\right)$ and $L^{2}(T)$, respectively, and on standard estimates of $L^{2}$-analysis for $B, h_{ \pm}, \mu_{ \pm}$of (3.15), (3.16), (3.17) and for the integral of (3.30).

Note that assumptions (3.23), (3.26), (3.29) of Proposition 3.1 are only some surplus sufficient conditions on $f$ for unique solvability of integral equations (3.12), (3.14), fulfilment of (3.30) and for boundedness of $A_{\mathrm{appr}}^{\mathrm{div}}, V_{\mathrm{appr}}^{\mathrm{div}}$.

Theorem 3.1. Let $f \in L^{2}\left(T^{2}\right)$ at fixed $E>0$. Suppose that $f$ satisfies (3.29) and is a smooth function on $T^{2}$ and $A_{\text {appr }}^{\text {div }}, V_{\text {appr }}^{\text {div }}$ are constructed from $f$ via the algorithm of Subsection (3.2). Then $A_{a p p r, 1}^{\text {div }}, A_{a p p r, 2}^{d i v}, V_{a p p r}^{\text {div }}$ are bounded functions on $\mathbb{R}^{2}$, decaying at infinity. In addition, $f$ is the scattering amplitude for equation (1.1) with $A=A_{a p p r}^{\text {div }}(x, E), V=V_{a p p r}^{\text {div }}(x, E)$.

For simplicity one can assume that $f \in C^{\infty}\left(T^{2}\right)$ in Theorem 3.1. However, very limited smoothness of $f$ is already sufficient. As regards to smoothness of $A_{\mathrm{appr}, 1}^{\mathrm{div}}, A_{\mathrm{appr}, 2}^{\mathrm{div}}, V_{\mathrm{appr}}^{\mathrm{div}}$ of Theorem 3.1 (which are complex-valued, in general), these functions are real-analytic functions of $x \in \mathbb{R}^{2}$. In addition, it is just for simplicity that we assume (3.29) in Theorem 3.1.

The proof of Theorem 3.1 is similar to the proof of Theorem 9.2 of [N3] for the case when $A \equiv 0$. Results of this type go back to [N1]. In the present work restrictions in time prevent us from proving Theorem 3.1 in details.

Finally, suppose that $f=f\left(\lambda, \lambda^{\prime}, E\right)$ is the scattering amplitude for equation (1.1) under assumptions (1.2) and that $A_{\mathrm{appr}}^{\text {div }}=A_{\mathrm{appr}}^{\text {div }}(x, E), V_{\mathrm{appr}}^{\text {div }}=V_{\mathrm{appr}}^{\text {div }}(x, E)$ are constructed from $f$ via the algorithm of Subsection 3.2 at fixed $E$. In the present work restrictions on time prevent us from obtaining estimates for $A^{\text {div }}$ $A_{\text {appr }}^{\text {div }}(\cdot, E)$ and $V^{\text {div }}-V_{\text {appr }}^{\text {div }}(\cdot, E)$ for sufficiently large $E$. For the linearized case such error estimates are given by formulas $(2.24),(2.25)$. For the nonlinearized case with $A \equiv 0$ such error estimates were given in [N4].

\section{Reduction of the nonlinearized reconstruction algorithm to inversion formulas of the Born ap- proximation}

Suppose that we are given $f$ on $M_{E} \cong T \times T=T^{2}$ at fixed E, where

$$
f \in L^{2}\left(T^{2}\right), \quad\|f\|_{L^{2}\left(T^{2}\right)} \leq \varepsilon .
$$


Proposition 4.1. Suppose that $f$ satisfies (4.1) at fixed $E>0$. Then, for $\varepsilon \rightarrow+0$, the nonlinearized reconstruction algorithm of Subsection 3.2 is reduced to the following formulas at fixed $E>0$ :

$$
\begin{gathered}
A_{a p p r, j}^{ \pm}(x, E)=\mathcal{A}_{a p p r, j}^{ \pm}(x, E)+O\left(\varepsilon^{2}\right), \quad j=1,2, \\
V_{a p p r}^{ \pm}(x, E)=\mathcal{V}_{a p p r}^{ \pm}(x, E)+O\left(\varepsilon^{2}\right), \\
A_{a p p r}^{d i v}(x, E)=\mathcal{A}_{a p p r, j}^{d i v}(x, E)+O\left(\varepsilon^{2}\right), \quad j=1,2, \\
V_{a p p r}^{d i v}(x, E)=\mathcal{V}_{a p p r}^{d i v}(x, E)+O\left(\varepsilon^{2}\right),
\end{gathered}
$$

where $O\left(\varepsilon^{2}\right)$ is considered in the uniform sense with respect to $x \in \mathbb{R}^{2}$ and where functions $\mathcal{A}_{\text {appr }, j}^{ \pm}, \mathcal{V}_{\text {appr }}^{ \pm}, j=1,2$, and $\mathcal{A}_{a p p r, j}^{\text {div }}, \mathcal{V}_{a p p r}^{\text {div }}, j=1,2$, are defined by the following linear formulas with respect to $f$ :

$$
\begin{gathered}
\mathcal{A}_{\text {appr }, 1}^{-}(x, E)=-\frac{i}{4} \sqrt{E} \int_{T^{2}} \operatorname{sgn}\left(\frac{1}{i}\left[\frac{\lambda}{\lambda^{\prime}}-\frac{\lambda^{\prime}}{\lambda}\right]\right)\left(\lambda-\lambda^{\prime}\right) f\left(\lambda, \lambda^{\prime}, z, E\right)|d \lambda|\left|d \lambda^{\prime}\right|, \\
\mathcal{A}_{\text {appr, }}^{-}(x, E)=-i \mathcal{A}_{\text {appr, }}^{-}(x, E), \\
\mathcal{V}_{a p p r}^{-}(x, E)=i \frac{E}{2} \int_{T^{2}}\left(1-\lambda \bar{\lambda}^{\prime}\right) \operatorname{sgn}\left(\frac{1}{i}\left[\frac{\lambda}{\lambda^{\prime}}-\frac{\lambda^{\prime}}{\lambda}\right]\right) f\left(\lambda, \lambda^{\prime}, z, E\right)|d \lambda|\left|d \lambda^{\prime}\right|, \\
\mathcal{A}_{a p p r, 1}^{+}(x, E)=\frac{i}{4} \sqrt{E} \int_{T^{2}} \operatorname{sgn}\left(\frac{1}{i}\left[\frac{\lambda}{\lambda^{\prime}}-\frac{\lambda^{\prime}}{\lambda}\right]\right) \overline{\left(\lambda-\lambda^{\prime}\right)} f\left(\lambda, \lambda^{\prime}, z, E\right)|d \lambda|\left|d \lambda^{\prime}\right|, \\
\mathcal{A}_{a p p r, 2}^{+}(x, E)=i \mathcal{A}_{a p p r, 1}^{+}(x, E), \\
\mathcal{A}_{a p p r, j}^{\text {div }}(x, E)=\frac{1}{2}\left(\mathcal{A}_{a p p r, j}^{+}(x, E)+\mathcal{A}_{a p p r, j}^{-}(x, E)\right), \quad j=1,2, \\
\mathcal{V}_{a p p r}^{\text {div }}(x, E)=\frac{E}{2} \int_{T^{2}}\left|\frac{1}{2 i}\left(\frac{\lambda}{T^{\prime}}-\frac{\lambda^{\prime}}{\lambda}\right)\right| f\left(\lambda, \lambda^{\prime}, z, E\right)|d \lambda|\left|d \lambda^{\prime}\right|,
\end{gathered}
$$

where

$$
\begin{gathered}
f\left(\lambda, \lambda^{\prime}, z, E\right) \stackrel{\text { def }}{=} f\left(\lambda, \lambda^{\prime}, E\right) \times \\
\times \exp \left(-i \frac{\sqrt{E}}{2}\left(\left(\lambda-\lambda^{\prime}\right) \bar{z}+\left(\lambda^{-1}-\lambda^{\prime-1}\right) z\right)\right),
\end{gathered}
$$

$\lambda \in T, \lambda^{\prime} \in T$ and $z, \bar{z}$ are given by (3.2).

Proposition 4.2. Suppose that $A_{1}, A_{2}, V \in C^{2, \sigma}\left(\mathbb{R}^{2}\right)$ for some $\sigma>2$, where $C^{N, \sigma}\left(\mathbb{R}^{2}\right)$ is defined by (2.12). Let $f^{\text {lin }}$ be defined by (2.2) and let $A_{\text {appr, }, \text { div }, 0}, V_{a p p r}^{\text {div }, 0}$, $A_{a p p r, j}^{ \pm, 0}, V_{a p p r}^{ \pm, 0}, j=1,2$, be defined by (2.21), (2.22), (2.23), (2.27), (2.28), (2.29) in terms of $f^{\text {lin }}$. Suppose also that functions $\mathcal{A}_{\text {appr }, j}^{\text {div }}, \mathcal{V}_{a p p r}^{\text {div }}, \mathcal{A}_{\text {appr }, j}^{ \pm}, \mathcal{V}_{a p p r}^{ \pm}, j=1$, 2 , are given by (4.6), (4.4), (4.5) with $f=f^{\text {lin }}$. Then the following equalities 
are valid:

$$
\begin{aligned}
A_{a p p r, j}^{d i v, 0}(x, E) & =\mathcal{A}_{a p p r, j}^{d i v}(x, E), \\
V_{a p p r}^{\text {div, }}(x, E) & =\mathcal{V}_{a p p r}^{d i v}(x, E), \\
A_{a p p r, j}^{ \pm, 0}(x, E) & =\mathcal{A}_{a p p r, j}^{ \pm}(x, E), \\
V_{a p p r}^{ \pm, 0}(x, E) & =\mathcal{V}_{a p p r}^{ \pm}(x, E),
\end{aligned}
$$

where $x \in \mathbb{R}^{2}, j=1,2, E>0$.

Propositions 4.1 and 4.2 are proved in Section 7.

\section{Proofs of Theorems 2.1, 2.2, 2.3}

Let us use the notations

$$
\widehat{u}(p)=(2 \pi)^{-2} \int_{\mathbb{R}^{2}} e^{i p x} u(x) d x, \quad u_{\mathrm{err}}(x, E)=\int_{|p| \geq 2 \sqrt{E}} e^{-i p x} \widehat{u}(p) d p,
$$

where $p \in \mathbb{R}^{2}, x \in \mathbb{R}^{2}, E>0$.

Lemma 5.1. Let $u \in C^{N, \sigma}\left(\mathbb{R}^{2}\right)$, where $N \geq 3, \sigma>2$. Then the following formula holds:

$$
\left|u_{e r r}(x, E)\right| \leq c_{1}(N, \sigma)\|u\|_{N, \sigma} E^{-\frac{N-2}{2}},
$$

where $x \in \mathbb{R}^{2}, E \geq 1 / 2$ and $c_{1}(N, \sigma)$ is defined by (2.20).

Proof of Lemma 5.1. We have that

$$
\widehat{\partial^{n} u}(p)=\left(-i p_{1}\right)^{n_{1}}\left(-i p_{2}\right)^{n_{2}} \widehat{u}(p),
$$

where $\partial^{n}$ is defined in (2.13), $p=\left(p_{1}, p_{2}\right) \in \mathbb{R}^{2}, n=\left(n_{1}, n_{2}\right) \in(\mathbb{N} \cup\{0\})^{2}$, $|n| \leq N$. Using this equality we obtain that

$$
|\widehat{u}(p)| \leq \frac{2^{N-1}}{\pi(\sigma-2)}\|u\|_{N, \sigma}\left(1+|p|^{2}\right)^{-\frac{N}{2}}
$$

for each $p \in \mathbb{R}^{2},|p| \geq 1$. Using the latter inequality we obtain (5.2).

Proof of Theorem 2.1. Since potentials $A$ and $V$ are real-valued it follows from (2.8) that the following formula holds:

$$
f^{\operatorname{lin}}(k, l)-\overline{f^{\operatorname{lin}}(l, k)}=2(k-l) \widehat{A}(k-l),
$$

where $(k, l) \in M_{E}$. We consider (2.8), (5.3) as a system of linear equations for finding $\widehat{A}(k-l)$ and $\widehat{V}(k-l)$. In addition, we use that $(k-l)(k+l)=0$ for $(k, l) \in M_{E}$, i.e. that vectors $(k-l)$ and $(k+l)$ are orthogonal. As a result we obtain formulas (2.15).

Formulas (2.16), (2.17) can be regarded as definitions of $A_{\text {appr }}, A_{\text {err }}, V_{\text {appr }}, V_{\text {err }}$. Estimates (2.18), (2.19) follow from Lemma 5.1 
Lemma 5.2. Suppose that $A_{1}, A_{2} \in C^{N, \sigma}\left(\mathbb{R}^{2}\right)$, where $N \geq 4, \sigma>2$. Let $\varphi^{\text {div }}$ be the solution of (2.4). Let $\widehat{\nabla \varphi}^{d i v},\left(\nabla \varphi^{d i v}\right)_{e r r}, \widehat{\Delta \varphi} d i v,\left(\Delta \varphi^{d i v}\right)_{e r r}$ be defined according to (5.1). Then the following estimates hold:

$$
\begin{aligned}
\left|\left(\partial_{j} \varphi^{d i v}\right)_{e r r}(x, E)\right| & \leq \sqrt{2} c_{1}(N, \sigma)\|A\|_{N, \sigma} E^{-\frac{N-2}{2}}, \\
\left|\left(\Delta \varphi^{d i v}\right)_{e r r}(x, E)\right| & \leq \sqrt{2} c_{1}(N, \sigma)\|A\|_{N, \sigma} E^{-\frac{N-3}{2}},
\end{aligned}
$$

where $x \in \mathbb{R}^{2}, j=1,2, E \geq 1 / 4,\|A\|_{N, \sigma}$ is defined by (2.26) and $c_{1}(N, \sigma)$ is defined by (2.20).

Proof of Lemma 5.2. The solution $\varphi^{\mathrm{div}}$ of (2.4) is given by:

$$
\varphi^{\operatorname{div}}(x)=-i \int_{\mathbb{R}^{2}} e^{-i p x}(p \widehat{A}(p))|p|^{-2} d p, \quad x \in \mathbb{R}^{2} .
$$

Using (5.1), (5.5) we obtain that

$$
\widehat{\nabla \varphi}^{\operatorname{div}}(p)=-p(p \widehat{A}(p))|p|^{-2}, \quad \widehat{\Delta \varphi}^{\operatorname{div}}(p)=i p \widehat{A}(p),
$$

where $p \in \mathbb{R}^{2} \backslash\{0\}$. Formulas (5.6) imply the following inequalities:

$$
\left|\widehat{\partial} j \varphi^{\operatorname{div}}(p)\right| \leq \sqrt{2} \max _{k=1,2}\left|\widehat{A}_{k}(p)\right|, \quad\left|\widehat{\Delta \varphi}^{\operatorname{div}}(p)\right| \leq \sqrt{2}|p| \max _{k=1,2}\left|\widehat{A}_{k}(p)\right|,
$$

where $p \in \mathbb{R}^{2} \backslash\{0\}, j=1,2$.

We have that

$$
\widehat{\partial^{n} A_{j}}(p)=\left(-i p_{1}\right)^{n_{1}}\left(-i p_{2}\right)^{n_{2}} \widehat{A}_{j}(p),
$$

where $p=\left(p_{1}, p_{2}\right) \in \mathbb{R}^{2}, n=\left(n_{1}, n_{2}\right) \in(\mathbb{N} \cup\{0\})^{2},|n| \leq N$. Using this equality we obtain that

$$
\left|\widehat{A}_{j}(p)\right| \leq \frac{2^{N-1}}{\pi(\sigma-2)}\left\|A_{j}\right\|_{N, \sigma}\left(1+|p|^{2}\right)^{-\frac{N}{2}}
$$

for $p \in \mathbb{R}^{2},|p| \geq 1, j=1,2$. Formulas (5.7), (5.8) imply the estimates

$$
\begin{aligned}
& \left|\widehat{\partial_{j} \varphi}{ }^{\operatorname{div}}(p)\right| \leq \sqrt{2} \frac{2^{N-1}}{\pi(\sigma-2)}\|A\|_{N, \sigma}\left(1+|p|^{2}\right)^{-\frac{N}{2}}, \\
& |\widehat{\Delta \varphi} \operatorname{div}(p)| \leq \sqrt{2} \frac{2^{N-1}}{\pi(\sigma-2)}\|A\|_{N, \sigma}\left(1+|p|^{2}\right)^{-\frac{N-1}{2}},
\end{aligned}
$$

where $p \in \mathbb{R}^{2},|p| \geq 1, j=1,2$. Using (5.9) we obtain (5.4).

Proof of Theorem 2.2. Taking into account invariance of $f^{\text {lin }}$ with respect to transformations (2.3) and using (2.4), (2.8) we obtain the following equalities:

$$
\begin{aligned}
(k-l) \widehat{A}^{\operatorname{div}, 0}(k-l) & =0, \\
f^{\operatorname{lin}}(k, l)-f^{\operatorname{lin}}(-l,-k) & =2(k+l) \widehat{A}^{\operatorname{div}, 0}(k-l), \\
f^{\operatorname{lin}}(k, l)+f^{\operatorname{lin}}(-l,-k) & =2 \widehat{V}^{\operatorname{div}, 0}(k-l),
\end{aligned}
$$


where $(k, l) \in M_{E}$. Using (5.10) and orthogonality of vectors $(k-l)$ and $(k+l)$ we obtain (2.21).

Formulas (2.22), (2.23) can be regarded as definitions of $A_{\text {appr }}^{\mathrm{div}, 0}, A_{\mathrm{err}}^{\mathrm{div}, 0}, V_{\mathrm{appr}}^{\mathrm{div}, 0}$, $V_{\text {err }}^{\text {div }, 0}$.

From (2.4), (2.22), (2.23) we derive

$$
\begin{aligned}
A_{\text {err }, \mathrm{j}}^{\mathrm{div}, 0}(x, E) & =\left(A_{j}\right)_{\mathrm{err}}(x, E)+\left(\partial_{j} \varphi^{\mathrm{div}}\right)_{\mathrm{err}}(x, E), \quad j=1,2, \\
V_{\mathrm{err}}^{\mathrm{div}, 0}(x, E) & =V_{\mathrm{err}}(x, E)-i\left(\Delta \varphi^{\mathrm{div}}\right)_{\mathrm{err}}(x, E),
\end{aligned}
$$

where $x \in \mathbb{R}^{2}, E>0$, and $\left(A_{1}\right)_{\text {err }},\left(A_{2}\right)_{\text {err }}, V_{\text {err }},\left(\nabla \varphi^{\text {div }}\right)_{\text {err }},\left(\Delta \varphi^{\text {div }}\right)_{\text {err }}$ are defined according to (5.1).

From (5.11) using inequalities (5.2) for $\left(A_{1}\right)_{\mathrm{err}},\left(A_{2}\right)_{\mathrm{err}}, V_{\mathrm{err}}$ and using inequalities (5.4) we obtain formulas (2.24), (2.25).

Lemma 5.3. Suppose that $A_{1}, A_{2} \in C^{N, \sigma}\left(\mathbb{R}^{2}\right)$, where $N \geq 4, \sigma>2$. Let $\varphi^{-}, \varphi^{+}$be the solutions of (2.5), (2.6), respectively. Let $\widehat{\nabla \varphi^{ \pm}},\left(\nabla \varphi^{ \pm}\right)_{e r r}, \widehat{\Delta \varphi^{ \pm}}$, $\left(\Delta \varphi^{ \pm}\right)_{\text {err }}$ be defined according to (5.1). Then the following estimates hold:

$$
\begin{aligned}
\left|\left(\partial_{j} \varphi^{ \pm}\right)_{e r r}(x, E)\right| & \leq \sqrt{2} c_{1}(N, \sigma)\|A\|_{N, \sigma} E^{-\frac{N-2}{2}}, \\
\left|\left(\Delta \varphi^{ \pm}\right)_{e r r}(x, E)\right| & \leq \sqrt{2} c_{1}(N, \sigma)\|A\|_{N, \sigma} E^{-\frac{N-3}{2}},
\end{aligned}
$$

where $x \in \mathbb{R}^{2}, j=1,2, E \geq 1 / 4,\|A\|_{N, \sigma}$ is defined by (2.26) and $c_{1}(N, \sigma)$ is defined by (2.20).

Proof of Lemma 2.3. The solutions $\varphi^{ \pm}$of (2.5), (2.6) are given by:

$$
\varphi^{ \pm}(x)=-i \int_{\mathbb{R}^{2}} e^{-i p x} \frac{\widehat{A}_{1}(p) \pm i \widehat{A}_{2}(p)}{p_{1} \pm i p_{2}} d p, \quad x \in \mathbb{R}^{2} .
$$

Using (5.1), (5.13) we obtain that

$$
\widehat{\nabla \varphi}^{ \pm}(p)=-\frac{\widehat{A}_{1}(p) \pm i \widehat{A}_{2}(p)}{p_{1} \pm i p_{2}} p, \quad \widehat{\Delta \varphi}^{ \pm}(p)=i \frac{\widehat{A}_{1}(p) \pm i \widehat{A}_{2}(p)}{p_{1} \pm i p_{2}}|p|^{2},
$$

where $p \in \mathbb{R}^{2} \backslash\{0\}$. Formulas (5.14) imply the following inequalities:

$$
\left|{\widehat{\partial_{j} \varphi}}^{ \pm}(p)\right| \leq \sqrt{2} \max _{k=1,2}\left|\widehat{A}_{k}(p)\right|, \quad\left|\widehat{\Delta \varphi}^{ \pm}(p)\right| \leq \sqrt{2}|p| \max _{k=1,2}\left|\widehat{A}_{k}(p)\right|,
$$

where $p \in \mathbb{R}^{2} \backslash\{0\}, j=1,2$.

As in the proof of Lemma 5.2 we have estimates (5.8). Formulas (5.8), (5.15) imply the following estimates:

$$
\begin{aligned}
& \left|\widehat{\partial_{j} \varphi^{ \pm}}(p)\right| \leq \sqrt{2} \frac{2^{N-1}}{\pi(\sigma-2)}\|A\|_{N, \sigma}\left(1+|p|^{2}\right)^{-\frac{N}{2}}, \\
& \left|\widehat{\Delta \varphi}^{ \pm}(p)\right| \leq \sqrt{2} \frac{2^{N-1}}{\pi(\sigma-2)}\|A\|_{N, \sigma}\left(1+|p|^{2}\right)^{-\frac{N-1}{2}},
\end{aligned}
$$

where $p \in \mathbb{R}^{2},|p| \geq 1, j=1,2$. Using (5.16) we obtain (5.12). 
Proof of Theorem 2.3. Taking into account invariance of $f^{\text {lin }}$ with respect to transformations (2.3) and using (2.5), (2.6), (2.8) we obtain the following equalities:

$$
\begin{aligned}
A_{2}^{ \pm, 0}(k-l) & = \pm i A_{1}^{ \pm, 0}(k-l), \\
f^{\operatorname{lin}}(k, l)-f^{\operatorname{lin}}(-l,-k) & =2\left(k_{1}+l_{1} \pm i\left(k_{2}+l_{2}\right)\right) \widehat{A}_{1}^{ \pm, 0}(k-l), \\
f^{\operatorname{lin}}(k, l)+f^{\operatorname{lin}}(-l,-k) & =2\left(k_{1}-l_{1} \pm i\left(k_{2}-l_{2}\right)\right) \widehat{A}_{1}^{ \pm, 0}(k-l)+2 \widehat{V}^{ \pm, 0}(k-l),
\end{aligned}
$$

where $(k, l) \in M_{E}$. Using (5.17) and orthogonality of vectors $(k-l)$ and $(k+l)$ we obtain (2.27). $V_{\mathrm{err}}^{ \pm 0}$.

Formulas (2.28), (2.29) can be regarded as definitions of $A_{\mathrm{appr}}^{ \pm, 0}, A_{\mathrm{err}}^{ \pm, 0}, V_{\mathrm{appr}}^{ \pm, 0}$,

From (2.5), (2.6), (2.28), (2.29) we derive formulas

$$
\begin{aligned}
A_{\text {err, }}^{ \pm, 0}(x, E) & =\left(A_{j}\right)_{\mathrm{err}}(x, E)+\left(\nabla \varphi^{ \pm}\right)_{\mathrm{err}}(x, E), \quad j=1,2, \\
V_{\text {err }}^{ \pm, 0}(x, E) & =V_{\mathrm{err}}(x, E)-i\left(\Delta \varphi^{ \pm}\right)_{\mathrm{err}}(x, E),
\end{aligned}
$$

where $x \in \mathbb{R}^{2}, E>0$, and $\left(A_{1}\right)_{\text {err }},\left(A_{2}\right)_{\text {err }}, V_{\text {err }},\left(\nabla \varphi^{ \pm}\right)_{\text {err }},\left(\Delta \varphi^{ \pm}\right)_{\text {err }}$ are defined by (5.1).

From (5.18) using inequalities (5.2) for $\left(A_{1}\right)_{\mathrm{err}},\left(A_{2}\right)_{\mathrm{err}}, V_{\mathrm{err}}$ and using inequalities (5.12) we obtain formulas (2.30), (2.31).

\section{Derivation of the reconstruction algorithm of Section 3}

6.1. Faddeev functions. For equation (1.1), under assumptions (1.2), we consider the Faddeev functions $\psi, h$ (see e.g. [F1], [F2] and subsection 5.1 of [HN]):

$$
\begin{gathered}
\psi(x, k)=e^{i k x} \mu(x, k), \\
\mu(x, k)=1+\int_{\mathbb{R}^{2}} g(x-y, k) \times \\
\times\left(-2 i A(y) \nabla_{y} \mu(y, k)+(2 A(y) k+V(y)) \mu(y, k)\right) d y, \\
g(x, k)=-(2 \pi)^{-2} \int_{\mathbb{R}^{2}} \frac{e^{i \xi x}}{\xi^{2}+2 k \xi} d \xi,
\end{gathered}
$$

where $x \in \mathbb{R}^{2}, k \in \Sigma_{E} \backslash \mathbb{R}^{2}$;

$$
\begin{gathered}
h(k, l)=(2 \pi)^{-2} \int_{\mathbb{R}^{2}} e^{i(k-l) y} \times \\
\times\left(-2 i A(y) \nabla_{y} \mu(y, k)+(2 A(y) k+V(y)) \mu(y, k)\right) d y,
\end{gathered}
$$

where $k, l \in \Sigma_{E} \backslash \mathbb{R}^{2}, \operatorname{Im} k=\operatorname{Im} l$. Here (6.1) and its differentiated version, where $\nabla$ is applied to both sides of (6.1), are considered as a system of linear integral equations for bounded $\mu$ and $\nabla \mu, \Sigma_{E}$ is defined by (3.4). 
We recall that $\psi$ are «growing» solutions of (1.1), in the sense of [F1], parametrized by $k \in \Sigma_{E} \backslash \mathbb{R}^{2}$, and $G=e^{i k x} g$ is the Faddeev's Green function for the operator $\Delta+k^{2}$.

Equation (6.1) for $\mu$ and formula (6.3) for $h$ are analogs in complex domain in $k$ of equation (1.6) for $\psi^{+}$and formula (1.8) for $f$.

Note that

$$
k, l \in \Sigma_{E} \backslash \mathbb{R}^{2}, \operatorname{Im} k=\operatorname{Im} l \Longrightarrow l=k \text { or } l=-\bar{k} .
$$

Therefore, the function $h$ of (6.3) splits to the functions

$$
a(k)=h(k, k), \quad b(k)=h(k,-\bar{k}), \quad k \in \Sigma_{E} \backslash \mathbb{R}^{2} .
$$

Note also that $a$ and $b$ are invariant with respect to transformations (1.10) and $\psi, \mu$ are transformed as

$$
\psi \rightarrow e^{-i \varphi} \psi, \quad \mu \rightarrow e^{-i \varphi} \mu
$$

with respect to (1.10).

6.2. Analytic properties of $\psi$. Using notations of Subsection 3.1 coefficients $A_{1}, A_{2}, V$ of equation (1.1), functions $\psi^{+}, f$ of (1.6), (1.8) and functions $\psi, \mu$, $b$ of (6.1), (6.5) can be written as

$$
\begin{gathered}
A_{1}=A_{1}(z), \quad A_{2}=A_{2}(z), \quad V=V(z), \\
\psi^{+}=\psi^{+}(z, \lambda, E), \quad f=f\left(\lambda, \lambda^{\prime}, E\right), \quad \lambda, \lambda^{\prime} \in T, \\
\psi=\psi(z, \lambda, E), \mu=\mu(z, \lambda, E), \quad b=b(\lambda, E), \quad \lambda \in \mathbb{C} \backslash(T \cup 0),
\end{gathered}
$$

where $z \in \mathbb{C}, E>0$.

It is known that the function $\psi$ (or $\mu$ ) has the following properties at fixed $z \in \mathbb{C}$ and $E>0$ (see page 448 of [N3]):

$$
\frac{\partial}{\partial \bar{\lambda}} \mu(z, \lambda, E)=r(\lambda, z, E) \mu\left(z,-\frac{1}{\bar{\lambda}}, E\right),
$$

for $\lambda \in \mathbb{C} \backslash(T \cup 0)$, where

$$
\begin{gathered}
r(\lambda, z, E)=\exp \left(-i \frac{\sqrt{E}}{2}\left(\lambda \bar{z}+\frac{z}{\lambda}+\bar{\lambda} z+\frac{\bar{z}}{\bar{\lambda}}\right)\right) \times \\
\times \frac{\pi}{\bar{\lambda}} \operatorname{sgn}(\lambda \bar{\lambda}-1) b(\lambda, E) ; \\
\mu(z, \lambda, E)=\mu_{0}^{-}(z)+o(1) \quad \text { for } \lambda \rightarrow \infty, \\
\mu(z, \lambda, E)=\mu_{0}^{+}(z)+o(1) \quad \text { for } \lambda \rightarrow 0,
\end{gathered}
$$

where

$$
\begin{gathered}
\partial_{\bar{z}} \mu_{0}^{+}(z)=\frac{1}{2 i}\left(A_{1}(z)+i A_{2}(z)\right) \mu_{0}^{+}(z), \\
\partial_{z} \mu_{0}^{-}(z)=\frac{1}{2 i}\left(A_{1}(z)-i A_{2}(z)\right) \mu_{0}^{-}(z), \\
\mu_{0}^{ \pm}(z) \rightarrow 1 \quad \text { as } z \rightarrow \infty ;
\end{gathered}
$$




$$
\begin{gathered}
\psi_{ \pm}(z, \lambda, E)=\psi^{+}(z, \lambda, E)+\pi i \int_{T} h_{ \pm}\left(\lambda, \lambda^{\prime \prime}, E\right) \chi\left( \pm i\left[\frac{\lambda}{\lambda^{\prime \prime}}-\frac{\lambda^{\prime \prime}}{\lambda}\right]\right) \times \\
\times \psi^{+}\left(z, \lambda^{\prime \prime}, E\right)\left|d \lambda^{\prime \prime}\right|
\end{gathered}
$$

for $\lambda \in T$, where

$$
\begin{aligned}
& \psi_{ \pm}(z, \lambda, E)=\psi(z, \lambda(1 \mp 0), E)=\exp \left(i \frac{\sqrt{E}}{2}\left(\lambda \bar{z}+\frac{z}{\lambda}\right)\right) \times \\
& \times \mu_{ \pm}(z, \lambda, E), \quad \mu_{ \pm}(z, \lambda, E)=\mu(z, \lambda(1 \pm 0), E), \quad \lambda \in T,
\end{aligned}
$$

$\psi^{+}$is the function of (1.6), (6.7), $h_{ \pm}$are the functions related with the scattering amplitude $f$ by equations (3.12), $\chi$ is defined by (3.13).

More precisely, equation (6.8) is fulfied if the system of linear equations for $\mu$ and $\nabla \mu$ related with (6.1) is uniquely solvable for $k=\left(k_{1}(\lambda, E), k_{2}(\lambda, E)\right)$ for fixed $\lambda \in \mathbb{C} \backslash(T \cup 0)$, where $k_{1}, k_{2}$ are given by (3.5), and relation (6.12) is fulfiled if the aforementioned system is uniquely solvable for $k=\left(k_{1}(\lambda)(1 \mp\right.$ $\left.0), E), k_{2}(\lambda(1 \mp 0), E)\right)$ for fixed $\lambda \in T$. In particular, all these conditions are fulfiled if coefficients $A_{1}, A_{2}, V$ of (1.1) are sufficiently small for fixed $E$.

6.3. Inverse scattering from $f$ and $b$. Using the definitions of $\varphi^{ \pm}, A^{ \pm}, V^{ \pm}$of (2.5), (2.6), (3.1), the invariance of $f$ and $b$ with respect to (1.10) and formulas (1.11), (6.6) one can see that

$$
\begin{aligned}
& \text { for } A, V \text { transformed to } A^{ \pm}, V^{ \pm} \text {(respectively) formulas } \\
& \text { (6.8)-(6.13) are fulfiled with } \mu_{0}^{ \pm} \equiv 1 \text { (respectively). }
\end{aligned}
$$

Properties (6.8)-(6.14) of $\psi, \mu$ yield the following approach to inverse scattering for equation (1.1) from $f$ and $b$ :

1. find $\psi, \mu$ satisfying $(6.8)-(6.10),(6.12),(6.13)$ with a priori unknown $\psi^{+}$ in $(6.12)$, where $\mu_{0}^{-} \equiv 1, \mu(z, \cdot, E) \in C(\mathbb{C} \backslash T), h_{ \pm}$are related with $f$ by $(3.12)$;

2. find $A^{-}, V^{-}$using that

$$
\begin{gathered}
A_{1}^{-}(z)-i A_{2}^{-}(z)=0, \quad A_{1}^{-}(z)+i A_{2}^{-}(z)=2 i \partial_{\bar{z}} \ln \mu_{0}^{+}(z), \\
V^{-}(z) \psi(z, \lambda, E)=\left(4 \partial_{z} \partial_{\bar{z}}+\right. \\
\left.+2 i\left(A_{1}^{-}(z)+i A_{2}^{-}(z)\right) \partial_{z}+E\right) \psi(z, \lambda, E),
\end{gathered}
$$

where $z \in \mathbb{C}, \lambda \in \mathbb{C} \backslash(T \cup 0)$.

Or alternatively:

1'. find $\psi, \mu$ satisfying (6.8)-(6.10), (6.12), (6.13) with a priori unknown $\psi^{+}$ in $(6.12)$, where $\mu_{0}^{+} \equiv 1, \mu(z, \cdot, E) \in C((\mathbb{C} \cup \infty) \backslash T), h_{ \pm}$are related with $f$ by (3.12); 
2'. find $A^{+}, V^{+}$using that

$$
\begin{gathered}
A_{1}^{+}(z)-i A_{2}^{+}(z)=2 i \partial_{z} \ln \mu_{0}^{-}(z), \quad A_{1}^{+}(z)+i A_{2}^{+}(z)=0, \\
V^{+}(z) \psi(z, \lambda, E)=\left(4 \partial_{z} \partial_{\bar{z}}+\right. \\
\left.+2 i\left(A_{1}^{+}(z)-i A_{2}^{+}(z)\right) \partial_{\bar{z}}+E\right) \psi(z, \lambda, E),
\end{gathered}
$$

where $z \in \mathbb{C}, \lambda \in \mathbb{C} \backslash(T \cup 0)$.

Note that (6.15) arises from (6.11) with $\mu_{0}^{-} \equiv 1,(6.17)$ arises from (6.11) with $\mu_{0}^{+} \equiv 1,(6.16)$ and (6.18) arise from equation (1.1) for the Faddeev functions $\psi$ of Subsections 6.1, 6.2 in the gauge setting related with $A^{-}, V^{-}$and $A^{+}, V^{+}$, respectively. In addition, $\psi, \mu, \mu_{0}^{+}$of steps 1,2 and $\psi=\psi^{\prime}, \mu=\mu^{\prime}, \mu_{0}^{-}$of steps $1^{\prime}, 2^{\prime}$ are related by the formulas

$$
\begin{gathered}
\psi^{\prime}(z, \lambda, E)=\left(\mu_{0}^{+}(z)\right)^{-1} \psi(z, \lambda, E), \\
\mu^{\prime}(z, \lambda, E)=\left(\mu_{0}^{+}(z)\right)^{-1} \mu(z, \lambda, E), \quad \mu_{0}^{-}(z)=\left(\mu_{0}^{+}(z)\right)^{-1},
\end{gathered}
$$

where $z \in \mathbb{C}, \lambda \in \mathbb{C} \backslash(T \cup 0)$.

As soon as $A, V$ are recovered as $A^{-}, V^{-}$or $A^{+}, V^{+}$, then these coefficients can be transformed from $A^{-}, V^{-}$or from $A^{+}, V^{+}$to other possible gauge setting via (1.10) and, in particular, to $A^{\text {div }}, V^{\text {div }}$ of (3.1).

Note that different ideas of the aforementioned approach to inverse scattering go back to $[\mathrm{M}],[\mathrm{ABF}]$, [GN1], [GM], [N3], [N4]. In particular, finding $\psi, \mu$ of the aforementioned steps 1 or $1^{\prime}$ for the case when $b \equiv 0$ at fixed $E$ is reduced to solving a non-local Riemann-Hilbert problem for holomorphic functions, see Subsection 6.4. Such non-local Riemann-Hilbert problems go back to [M].

6.4. Inverse scattering with $b \equiv 0$ at fixed $E$. In the Born approximation at fixed $E$ we have that

$$
\begin{array}{rlrl}
f(k, l) & \approx f^{\operatorname{lin}}(k, l)=2 k \widehat{A}(k-l)+\widehat{V}(k-l), & & (k, l) \in M_{E}, \\
b(k) & \approx b^{\operatorname{lin}}(k)=2 k \widehat{A}(2 \operatorname{Re} k)+\widehat{V}(2 \operatorname{Re} k), & k \in \Sigma_{E} \backslash \mathbb{R}^{2},
\end{array}
$$

where $\widehat{A}, \widehat{V}$ are defined by (2.9). Here formula (6.20) is equivalent to the formulas for $f$ of (2.1), (2.2) and formula (6.21) follows from (6.1), (6.3), (6.5) in a similar way that (6.20) follows from (1.6), (1.8). Note also that

$$
k \in \Sigma_{E} \backslash \mathbb{R}^{2} \Longrightarrow 2 \operatorname{Re} k \in \mathbb{R}^{2} \backslash B_{2 \sqrt{E}}, \quad E>0,
$$

where $B_{r}$ is defined by $(2.11)$.

Using (2.10), (6.20) and (6.21), (6.22) one can see that the expression for $f^{\text {lin }}$ involves $\widehat{A}, \widehat{V}$ on $B_{2 \sqrt{E}}$, only, and the expression for $b^{\text {lin }}$ involves $\widehat{A}, \widehat{V}$ on $\mathbb{R}^{2} \backslash B_{2 \sqrt{E}}$, only, at fixed $E$. Further, using also (2.21)-(2.23), (2.27)-(2.29) one can see that the expressions for $A_{\text {appr }}^{ \pm, 0}, V_{\text {appr }}^{ \pm, 0}, A_{\text {appr }}^{\text {div, },}, V_{\text {appr }}^{\text {div }, 0}$ involve $f^{\text {lin }}$, only, and are independent of $b^{\text {lin }}$ at fixed $E$.

In a similar way, in Section 3 in order to construct nonlinear analogs of $A_{\text {appr }}^{ \pm, 0}, V_{\text {appr }}^{ \pm, 0}, A_{\text {appr }}^{\text {div, },}, V_{\text {appr }}^{\text {div, }, 0}$ we use inverse scattering of Subsection 6.3 without $b$ or, in other words, with $b \equiv 0$ at fixed $E$. 
In this case steps 1 and 1' of Subsection 6.3 consist in solving the following non-local Riemann-Hilbert problems for holomorphic functions:

1. find $\psi=\exp ((i / 2) \sqrt{E}(\lambda \bar{z}+z / \lambda)) \mu(z, \lambda, E), z \in \mathbb{C}, \lambda \in \mathbb{C} \backslash(T \cup 0)$, satisfying $(6.12),(6.13)$ with a priori unknown $\psi^{+}$in $(6.12)$, where

$$
\begin{gathered}
\frac{\partial}{\partial \bar{\lambda}} \mu(z, \lambda, E)=0, \quad \lambda \in \mathbb{C} \backslash(T \cup 0), \\
\mu(z, \lambda, E) \rightarrow 1, \quad \text { as } \lambda \rightarrow \infty, \\
\mu(z, \cdot, E) \in C(\mathbb{C} \backslash T),
\end{gathered}
$$

or, alternatively:

1'. find $\psi=\exp ((i / 2) \sqrt{E}(\lambda \bar{z}+z / \lambda)) \mu(z, \lambda, E), z \in \mathbb{C}, \lambda \in \mathbb{C} \backslash(T \cup 0)$, satisfying (6.12), (6.13) with a propri unknown $\psi^{+}$in $(6.12)$, where

$$
\begin{gathered}
\frac{\partial}{\partial \bar{\lambda}} \mu(z, \lambda, E)=0, \quad \lambda \in \mathbb{C} \backslash(T \cup 0), \\
\mu(z, \lambda, E) \rightarrow 1, \quad \text { as } \lambda \rightarrow 0, \\
\mu(z, \cdot, E) \in C((\mathbb{C} \cup \infty) \backslash T) .
\end{gathered}
$$

We recall that $h_{ \pm}$of (6.12) are related with $f$ by (3.12). Actually, it is also assumed that $\mu(z, \cdot, E)$ admits continuous extension on $T$ from its both sides.

Now due to considerations of Section 2 of [N4] we have that finding $\mu$ of step 1 is reduced to: (a) solving the linear integral equation (3.14) for $\mu^{+}(z, \cdot, E)$ on $T$, where $\psi^{+}=\exp ((i / 2) \sqrt{E}(\lambda \bar{z}+z / \lambda)) \mu^{+}(z, \lambda, E), z \in \mathbb{C}, \lambda \in T$, (b) finding $\mu_{ \pm}(z, \cdot, E)$ on $T$ by formulas (3.17) (or, in other words, by formulas (6.12) rewritten in terms of $\mu_{ \pm}$and $\mu^{+}$), (c) finding $\mu(z, \cdot, E)$ on $C \backslash T$ by the Cauchy formulas:

$$
\begin{aligned}
& \mu(z, \lambda, E)=\frac{1}{2 \pi i} \int_{T} \frac{\mu_{+}(z, \zeta, E)}{\zeta-\lambda} d \zeta, \quad|\lambda|<1, \\
& \mu(z, \lambda, E)=1-\frac{1}{2 \pi i} \int_{T} \frac{\mu_{-}(z, \zeta, E)}{\zeta-\lambda} d \zeta, \quad|\lambda|>1,
\end{aligned}
$$

$z \in \mathbb{C}, \lambda \in \mathbb{C} \backslash T$.

In addition, due to (6.19), (6.25), finding $\psi=\psi^{\prime}, \mu=\mu^{\prime}$ of step 1 ' is reduced to finding $\psi, \mu$ of step 1 and to the formulas

$$
\begin{gathered}
\psi^{\prime}(z, \lambda, E)=\left(\mu_{0}^{+}(z)\right)^{-1} \psi(z, \lambda, E), \quad \mu^{\prime}(z, \lambda, E)=\left(\mu_{0}^{+}(z)\right)^{-1} \mu(z, \lambda, E), \\
\mu_{0}^{+}(z)=\frac{1}{2 \pi i} \int_{T} \frac{\mu_{+}(z, \zeta, E)}{\zeta} d \zeta
\end{gathered}
$$

where $z \in \mathbb{C}, \lambda \in \mathbb{C} \backslash(T \cup 0)$. 
In addition, due to $(6.25)$ we have that

$$
\begin{aligned}
& \mu(z, \lambda, E)=1+\mu_{1}^{-}(z) \lambda^{-1}+O\left(\lambda^{-2}\right) \quad \text { as } \lambda \rightarrow \infty, \\
& \mu(z, \lambda, E)=\mu_{0}^{+}(z)+\mu_{1}^{+}(z) \lambda+O\left(\lambda^{2}\right) \quad \text { as } \lambda \rightarrow 0,
\end{aligned}
$$

where $z \in \mathbb{C}, \lambda \in \mathbb{C} \backslash(T \cup 0), \mu_{0}^{+}(z)$ is given in (6.26),

$$
\begin{aligned}
& \mu_{1}^{+}(z)=\frac{1}{2 \pi i} \int_{T} \frac{\mu_{+}(z, \zeta, E)}{\zeta^{2}} d \zeta, \\
& \mu_{1}^{-}(z)=\frac{1}{2 \pi i} \int_{T} \mu_{-}(z, \zeta, E) d \zeta
\end{aligned}
$$

Note that the non-local Riemann-Hilbert problems of steps 1 and 1' are better known in the literature (going back to $[\mathrm{M}]$ ) for the case when relation (6.12) between $\psi_{+}$and $\psi_{-}$on $T$ with a priori unknown $\psi^{+}$is given in the form

$$
\psi_{+}(\lambda)=\psi_{-}(\lambda)+\int_{T} \rho\left(\lambda, \lambda^{\prime}\right) \psi_{-}\left(\lambda^{\prime}\right)\left|d \lambda^{\prime}\right|, \quad \lambda \in T,
$$

see e.g. [M], [GN1], [GM], [N3]. Note also that in our case $h_{ \pm}$of (3.12), (6.12) are related with $\rho$ of (6.29) by the following formulas and equations:

$$
\begin{aligned}
h_{1}\left(\lambda, \lambda^{\prime}, E\right)= & \chi\left(i\left[\frac{\lambda^{\prime}}{\lambda}-\frac{\lambda}{\lambda^{\prime}}\right]\right) h_{+}\left(\lambda, \lambda^{\prime}, E\right)- \\
& -\chi\left(-i\left[\frac{\lambda^{\prime}}{\lambda}-\frac{\lambda}{\lambda^{\prime}}\right]\right) h_{-}\left(\lambda, \lambda^{\prime}, E\right), \\
h_{2}\left(\lambda, \lambda^{\prime}, E\right)= & \chi\left(i\left[\frac{\lambda^{\prime}}{\lambda}-\frac{\lambda}{\lambda^{\prime}}\right]\right) h_{-}\left(\lambda, \lambda^{\prime}, E\right)- \\
& -\chi\left(-i\left[\frac{\lambda^{\prime}}{\lambda}-\frac{\lambda}{\lambda^{\prime}}\right]\right) h_{+}\left(\lambda, \lambda^{\prime}, E\right), \\
\rho\left(\lambda, \lambda^{\prime}, E\right)+ & \pi i \int_{T} \rho\left(\lambda, \lambda^{\prime \prime}, E\right) \chi\left(-i\left[\frac{\lambda^{\prime}}{\lambda^{\prime \prime}}-\frac{\lambda^{\prime \prime}}{\lambda^{\prime}}\right]\right) \times \\
& \times h_{1}\left(\lambda^{\prime \prime}, \lambda^{\prime}, E\right)\left|d \lambda^{\prime \prime}\right|=-\pi i h_{1}\left(\lambda, \lambda^{\prime}, E\right), \\
\rho\left(\lambda, \lambda^{\prime}, E\right)+ & \pi i \int_{T} \chi\left(i\left[\frac{\lambda^{\prime}}{\lambda^{\prime \prime}}-\frac{\lambda^{\prime \prime}}{\lambda^{\prime}}\right]\right) \times \\
& \times h_{2}\left(\lambda^{\prime \prime}, \lambda^{\prime}, E\right)\left|d \lambda^{\prime \prime}\right|=-\pi i h_{2}\left(\lambda, \lambda^{\prime}, E\right),
\end{aligned}
$$

where $\lambda, \lambda^{\prime} \in T$, see [N3].

Futher, due to results of [GN1], [N3] and of Proposition 3.1 of the present work we have that, at least under assumption (3.26), the non-local RiemannHilbert problems for $\psi$ and for $\psi=\psi^{\prime}$ of steps 1 and $1^{\prime}$ are uniquely solvable 
and

$$
\begin{array}{r}
\left(-4 \partial_{z} \partial_{\bar{z}}+a_{z}^{-}(z) \partial_{z}+V^{-}(z)\right) \psi(z, \lambda, E)=E \psi(z, \lambda, E), \\
a_{z}^{-}(z)=4 \partial_{\bar{z}} \ln \mu_{0}^{+}(z), \quad V^{-}(z)=2 i \sqrt{E} \partial_{z} \mu_{1}^{-}(z), \\
\left(-4 \partial_{z} \partial_{\bar{z}}+a_{\bar{z}}^{+}(z) \partial_{\bar{z}}+V^{+}(z)\right) \psi^{\prime}(z, \lambda, E)=E \psi^{\prime}(z, \lambda, E), \\
a_{\bar{z}}^{+}(z)=4 \partial_{z} \ln \frac{1}{\mu_{0}^{+}(z)}, \quad V^{+}(z)=2 i \sqrt{E} \partial_{\bar{z}} \frac{\mu_{1}^{+}(z)}{\mu_{0}^{+}(z)}
\end{array}
$$

where $z \in \mathbb{C}, \lambda \in \mathbb{C} \backslash(T \cup 0), \mu_{0}^{+}, \mu_{1}^{-}, \mu_{1}^{+}$are the coefficients of (6.27). Here (6.32), (6.33) correspond to (6.15), (6.16) and (6.34), (6.35) correspond to (6.17), (6.18).

Formulas (3.18), (3.19) follow from (6.32)-(6.35) and the integral expression for $\mu_{0}^{+}, \mu_{1}^{-}, \mu_{1}^{+}$of $(6.26),(6.28)$.

Finally, formulas (3.20) arise from considerations of the gauge transformations (1.10) between $A^{-}, V^{-}$and $A^{+}, V^{+}$and $A^{\text {div }}, V^{\text {div }}$ of (3.1). In particular, in these considerations we use that for equation (1.1) written as

$$
\begin{gathered}
\left(-4 \partial_{z} \partial_{\bar{z}}+a_{z} \partial_{z}+a_{\bar{z}} \partial_{\bar{z}}+V\right) \psi=E \psi, \\
a_{z}=-2 i\left(A_{1}+i A_{2}\right), \quad a_{\bar{z}}=-2 i\left(A_{1}-i A_{2}\right),
\end{gathered}
$$

the gauge transformations $(1.10),(1.11)$ can be written as

$$
\begin{aligned}
a_{z} & \rightarrow a_{z}-4 i \partial_{\bar{z}} \varphi, \quad a_{\bar{z}} \rightarrow a_{\bar{z}}-4 i \partial_{z} \varphi \\
V & \rightarrow V-4 i \partial_{z} \partial_{\bar{z}} \varphi+4 \partial_{z} \varphi \partial_{\bar{z}} \varphi+i a_{z} \partial_{z} \varphi+i a_{\bar{z}} \partial_{\bar{z}} \varphi \\
\psi & \rightarrow e^{-i \varphi} \psi
\end{aligned}
$$

and that the equations for $\varphi^{\operatorname{div}}, \varphi^{-}, \varphi^{+}$of $(2.4)-(2.6),(3.1)$ can be written as

$$
\begin{gathered}
8 i \partial_{z} \partial_{\bar{z}} \varphi^{\operatorname{div}}=\partial_{z} a_{z}+\partial_{\bar{z}} a_{\bar{z}}, \quad \varphi^{\operatorname{div}}(z) \rightarrow 0 \text { as }|z| \rightarrow \infty \\
4 i \partial_{z} \varphi^{-}=a_{\bar{z}}, \quad \varphi^{-}(z) \rightarrow 0 \text { as }|z| \rightarrow \infty \\
4 i \partial_{\bar{z}} \varphi^{+}=a_{z}, \quad \varphi^{+}(z) \rightarrow 0 \text { as }|z| \rightarrow \infty
\end{gathered}
$$

\section{Proofs of Propositions 4.1 and 4.2}

Proof of Proposition 4.1. The method of successive approximations for solving (3.12) with repsect to $h_{ \pm} \in L^{2}\left(T^{2}\right)$ and assumptions (4.1) imply that

$$
h_{ \pm}=f+O\left(\varepsilon^{2}\right), \quad \varepsilon \rightarrow+0
$$

where $O\left(\varepsilon^{2}\right)$ is considered in the sense of $\|\cdot\|_{L^{2}\left(T^{2}\right)}$.

Consider the following operators acting in $L^{2}(T)$

$$
\left(C_{ \pm} u\right)(\lambda)=\frac{1}{2 \pi i} \int_{T} \frac{u(\zeta)}{\zeta-\lambda(1 \mp 0)} d \zeta, \quad \lambda \in T
$$


where $u$ is a test function. We recall that

$$
\left\|C_{ \pm} u\right\|_{L^{2}(T)} \leq\|u\|_{L^{2}(T)} .
$$

Using (3.15), (3.16), (7.1), (7.3) and the equality

$$
\begin{gathered}
\left|\exp \left(-i \frac{\sqrt{E}}{2}\left(\left(\lambda-\lambda^{\prime}\right) \bar{z}+\left(\lambda^{-1}-\lambda^{\prime-1}\right) z\right)\right)\right|=1, \\
\lambda, \lambda^{\prime} \in T, \quad z \in \mathbb{C}, \quad E>0
\end{gathered}
$$

we obtain that

$$
\begin{gathered}
B\left(\lambda, \lambda^{\prime}, z, E\right)=\frac{1}{2} \int_{T} f\left(\zeta, \lambda^{\prime}, z, E\right) \chi\left(-i\left[\frac{\zeta}{\lambda^{\prime}}-\frac{\lambda^{\prime}}{\zeta}\right]\right) \frac{d \zeta}{\zeta-\lambda(1-0)}- \\
-\frac{1}{2} \int_{T} f\left(\zeta, \lambda^{\prime}, z, E\right) \chi\left(i\left[\frac{\zeta}{\lambda^{\prime}}-\frac{\lambda^{\prime}}{\zeta}\right]\right) \frac{d \zeta}{\zeta-\lambda(1+0)}+O\left(\varepsilon^{2}\right),
\end{gathered}
$$

where $\lambda, \lambda^{\prime} \in T, z \in \mathbb{C}, f\left(\zeta, \lambda^{\prime}, z, E\right)$ is given by (4.7), $O\left(\varepsilon^{2}\right)$ is considered in the sense of $\|\cdot\|_{L^{2}\left(T^{2}\right)}$ and is uniform with respect to $z \in \mathbb{C}$.

From (3.14) we derive the following equalities:

$$
\begin{gathered}
\partial_{z} \mu^{+}(z, \lambda, E)+\int_{T} B\left(\lambda, \lambda^{\prime}, z, E\right) \partial_{z} \mu^{+}\left(z, \lambda^{\prime}, E\right)\left|d \lambda^{\prime}\right|= \\
=-\int_{T} \partial_{z} B\left(\lambda, \lambda^{\prime}, z, E\right) \mu^{+}\left(z, \lambda^{\prime}, E\right)\left|d \lambda^{\prime}\right|, \\
\partial_{\bar{z}} \mu^{+}(z, \lambda, E)+\int_{T} B\left(\lambda, \lambda^{\prime}, z, E\right) \partial_{\bar{z}} \mu^{+}\left(z, \lambda^{\prime}, E\right)\left|d \lambda^{\prime}\right|= \\
=-\int_{T} \partial_{\bar{z}} B\left(\lambda, \lambda^{\prime}, z, E\right) \mu^{+}\left(z, \lambda^{\prime}, E\right)\left|d \lambda^{\prime}\right|,
\end{gathered}
$$

where $\lambda \in T, z \in \mathbb{C}, E>0$.

The method of successive approximations for solving (3.14) and (7.6) with respect to $\mu_{+} \in L^{2}(T)$ and $\partial_{z} \mu_{+}, \partial_{\bar{z}} \mu_{+} \in L^{2}(T)$ and estimates (4.1), (7.5) imply that:

$$
\begin{aligned}
\mu^{+}(z, \lambda, E) & =1+O(\varepsilon), \\
\partial_{z} \mu^{+}(z, \lambda, E) & =-\int_{T} \partial_{z} B\left(\lambda, \lambda^{\prime}, z, E\right)\left|d \lambda^{\prime}\right|+O\left(\varepsilon^{2}\right), \\
\partial_{\bar{z}} \mu^{+}(z, \lambda, E) & =-\int_{T} \partial_{\bar{z}} B\left(\lambda, \lambda^{\prime}, z, E\right)\left|d \lambda^{\prime}\right|+O\left(\varepsilon^{2}\right),
\end{aligned}
$$

where $z \in \mathbb{C}, \lambda \in T, O(\varepsilon), O\left(\varepsilon^{2}\right)$ are considered in the sense of $\|\cdot\|_{L^{2}(T)}$ and are uniform with respect to $z \in \mathbb{C}$. 
Using (3.17), (7.1), (7.7) we obtain that

$$
\begin{aligned}
\mu_{ \pm}(z, \lambda, E) & =1+O(\varepsilon), \\
\partial_{z} \mu_{ \pm}(z, \lambda, E) & =-\int_{T} \partial_{z} B\left(\lambda, \lambda^{\prime}, z, E\right)\left|d \lambda^{\prime}\right|+ \\
& +\pi i \int_{T} \partial_{z} f\left(\lambda, \lambda^{\prime}, z, E\right) \chi\left( \pm i\left[\frac{\lambda}{\lambda^{\prime}}-\frac{\lambda^{\prime}}{\lambda}\right]\right)\left|d \lambda^{\prime}\right|+O\left(\varepsilon^{2}\right), \\
\partial_{\bar{z}} \mu_{ \pm}(z, \lambda, E) & =-\int_{T} \partial_{\bar{z}} B\left(\lambda, \lambda^{\prime}, z, E\right)\left|d \lambda^{\prime}\right|+ \\
& +\pi i \int_{T} \partial_{\bar{z}} f\left(\lambda, \lambda^{\prime}, z, E\right) \chi\left( \pm i\left[\frac{\lambda}{\lambda^{\prime}}-\frac{\lambda^{\prime}}{\lambda}\right]\right)\left|d \lambda^{\prime}\right|+O\left(\varepsilon^{2}\right),
\end{aligned}
$$

where $\lambda \in T, f\left(\lambda, \lambda^{\prime}, z, E\right)$ is given by (4.7) and $O(\varepsilon), O\left(\varepsilon^{2}\right)$ are considered in the sense of $\|\cdot\|_{L^{2}(T)}$ and are uniform with repsect to $z \in \mathbb{C}$.

Using (3.18), (3.19), (7.8) we obtain that:

$$
\begin{aligned}
V_{\mathrm{appr}}^{-}(x, E) & =-\frac{\sqrt{E}}{\pi} \int_{T^{2}} \partial_{z} B\left(\lambda, \lambda^{\prime}, z, E\right) d \lambda\left|d \lambda^{\prime}\right|+ \\
& +i \sqrt{E} \int_{T^{2}} \partial_{z} f\left(\lambda, \lambda^{\prime}, z, E\right) \chi\left(-i\left[\frac{\lambda}{\lambda^{\prime}}-\frac{\lambda^{\prime}}{\lambda}\right]\right) d \lambda\left|d \lambda^{\prime}\right|+O\left(\varepsilon^{2}\right) \\
V_{\mathrm{appr}}^{+}(x, E) & =\frac{\sqrt{E}}{\pi} \int_{T^{2}} \partial_{\bar{z}} B\left(\lambda, \lambda^{\prime}, z, E\right) \lambda^{-2} d \lambda\left|d \lambda^{\prime}\right|- \\
& -i \sqrt{E} \int_{T^{2}} \partial_{\bar{z}} f\left(\lambda, \lambda^{\prime}, z, E\right) \chi\left(i\left[\frac{\lambda}{\lambda^{\prime}}-\frac{\lambda^{\prime}}{\lambda}\right]\right) \lambda^{-2} d \lambda\left|d \lambda^{\prime}\right|+O\left(\varepsilon^{2}\right), \\
a_{z}^{-}(z, E) & =\frac{2 i}{\pi} \int_{T^{2}} \partial_{\bar{z}} B\left(\lambda, \lambda^{\prime}, z, E\right) \lambda^{-1} d \lambda\left|d \lambda^{\prime}\right|+ \\
& +2 \int_{T^{2}} \partial_{\bar{z}} f\left(\lambda, \lambda^{\prime}, z, E\right) \chi\left(i\left[\frac{\lambda}{\lambda^{\prime}}-\frac{\lambda^{\prime}}{\lambda}\right]\right) \lambda^{-1} d \lambda\left|d \lambda^{\prime}\right|+O\left(\varepsilon^{2}\right) \\
a_{\bar{z}}^{+}(z, E) & =-\frac{2 i}{\pi} \int_{T^{2}} \partial_{z} B\left(\lambda, \lambda^{\prime}, z, E\right) \lambda^{-1} d \lambda\left|d \lambda^{\prime}\right|- \\
& -2 \int_{T^{2}} \partial_{z} f\left(\lambda, \lambda^{\prime}, z, E\right) \chi\left(i\left[\frac{\lambda}{\lambda^{\prime}}-\frac{\lambda^{\prime}}{\lambda}\right]\right) \lambda^{-1} d \lambda\left|d \lambda^{\prime}\right|+O\left(\varepsilon^{2}\right),
\end{aligned}
$$

where $x \in \mathbb{R}^{2}, z$ is given by (3.2), and $O\left(\varepsilon^{2}\right)$ is considered in the uniform sense with respect to $x \in \mathbb{R}^{2}$. 
Note that the following formulas hold for each $u \in L^{2}(T)$ :

$$
\begin{aligned}
& \int_{T}\left(C_{+} u\right)(\lambda) d \lambda=0, \quad \int_{T}\left(C_{-} u\right)(\lambda) d \lambda=-\int_{T} u(\lambda) d \lambda, \\
& \int_{T}\left(C_{+} u\right)(\lambda) \frac{d \lambda}{\lambda}=\int_{T} u(\lambda) \frac{d \lambda}{\lambda}, \quad \int_{T}\left(C_{-} u\right)(\lambda) \frac{d \lambda}{\lambda}=0, \\
& \int_{T}\left(C_{+} u\right)(\lambda) \frac{d \lambda}{\lambda^{2}}=\int_{T} u(\lambda) \frac{d \lambda}{\lambda^{2}}, \quad \int_{T}\left(C_{-} u\right)(\lambda) \frac{d \lambda}{\lambda^{2}}=0,
\end{aligned}
$$

where $C_{ \pm}$are defined by (7.2).

Formulas (7.5), (7.9), (7.10) imply estimates

$$
\begin{aligned}
& V_{\text {appr }}^{-}(x, E)=i \sqrt{E} \int_{T^{2}} s\left(\lambda, \lambda^{\prime}\right) \partial_{z} f\left(\lambda, \lambda^{\prime}, z, E\right) d \lambda\left|d \lambda^{\prime}\right|+O\left(\varepsilon^{2}\right), \\
& V_{\text {appr }}^{+}(x, E)=i \sqrt{E} \int_{T^{2}} s\left(\lambda, \lambda^{\prime}\right) \partial_{\bar{z}} f\left(\lambda, \lambda^{\prime}, z, E\right) \lambda^{-2} d \lambda\left|d \lambda^{\prime}\right|+O\left(\varepsilon^{2}\right), \\
& a_{z}^{-}(z, E)=-2 \int_{T^{2}} s\left(\lambda, \lambda^{\prime}\right) \partial_{\bar{z}} f\left(\lambda, \lambda^{\prime}, z, E\right) \lambda^{-1} d \lambda\left|d \lambda^{\prime}\right|+O\left(\varepsilon^{2}\right), \\
& a_{z}^{-}(z, E)=2 \int_{T^{2}} s\left(\lambda, \lambda^{\prime}\right) \partial_{z} f\left(\lambda, \lambda^{\prime}, z, E\right) \lambda^{-1} d \lambda\left|d \lambda^{\prime}\right|+O\left(\varepsilon^{2}\right), \\
& s\left(\lambda, \lambda^{\prime}\right) \stackrel{\text { def }}{=} \operatorname{sgn}\left(\frac{1}{i}\left[\frac{\lambda}{\lambda^{\prime}}-\frac{\lambda^{\prime}}{\lambda}\right]\right),
\end{aligned}
$$

where $x \in \mathbb{R}^{2}, z$ is given by (3.2), and $O\left(\varepsilon^{2}\right)$ is considered in the uniform sense with respect to $x \in \mathbb{R}^{2}$.

In addition, due to (4.7) we have that

$$
\begin{aligned}
& \partial_{z} f\left(\lambda, \lambda^{\prime}, z, E\right)=-i \frac{\sqrt{E}}{2}\left(\lambda^{-1}-\lambda^{\prime-1}\right) f\left(\lambda, \lambda^{\prime}, z, E\right), \\
& \partial_{\bar{z}} f\left(\lambda, \lambda^{\prime}, z, E\right)=-i \frac{\sqrt{E}}{2}\left(\lambda-\lambda^{\prime}\right) f\left(\lambda, \lambda^{\prime}, z, E\right),
\end{aligned}
$$

for each $\lambda, \lambda^{\prime} \in T, z \in \mathbb{C}, E>0$.

Formulas (4.2), (4.3), (4.4), (4.5), (4.6) follow immediately from (3.18), (3.19), (3.20), (7.11), (7.12).

Proposition 4.1 is proved.

Lemma 7.1. Let $E>0$ be fixed. Let $u\left(\lambda, \lambda^{\prime}, E\right),\left(\lambda, \lambda^{\prime}\right) \in T^{2}$, be a complex valued function such that $u \in L^{1}\left(T^{2}\right)$ and

$$
u\left(\lambda, \lambda^{\prime}, E\right)=u\left(-\lambda^{\prime},-\lambda, E\right), \quad\left(\lambda, \lambda^{\prime}\right) \in T^{2} .
$$


Suppose that $g(p, E), p=\left(p_{1}, p_{2}\right) \in \mathbb{R}^{2},|p| \leq 2 \sqrt{E}$, is the function defined by the formula

$$
g\left(\sqrt{E} \operatorname{Re}\left(\lambda-\lambda^{\prime}\right), \sqrt{E} \operatorname{Im}\left(\lambda-\lambda^{\prime}\right), E\right)=u\left(\lambda, \lambda^{\prime}, E\right),
$$

where $\left(\lambda, \lambda^{\prime}\right) \in T^{2}$. Then

$$
\begin{gathered}
\int_{|p| \leq 2 \sqrt{E}} e^{-i p x} g(p, E) d p=\frac{E}{2} \int_{T^{2}} u\left(\lambda, \lambda^{\prime}, z, E\right)\left|\frac{1}{2 i}\left(\frac{\lambda}{\lambda^{\prime}}-\frac{\lambda^{\prime}}{\lambda}\right)\right||d \lambda|\left|d \lambda^{\prime}\right|, \\
u\left(\lambda, \lambda^{\prime}, z, E\right) \stackrel{\text { def }}{=} u\left(\lambda, \lambda^{\prime}, E\right) \times \\
\times \exp \left(-i \frac{\sqrt{E}}{2}\left(\left(\lambda-\lambda^{\prime}\right) \bar{z}+\left(\lambda^{-1}-\lambda^{\prime-1}\right) z\right)\right),
\end{gathered}
$$

where $x \in \mathbb{R}^{2}$, $z$ is given by (3.2).

Actually, (7.15) arises from the following change of variables in the integration with respect to $p$ :

$$
\begin{aligned}
& p_{1}=\sqrt{E} \operatorname{Re}\left(\lambda-\lambda^{\prime}\right)=\sqrt{E}\left(\cos \phi-\cos \phi^{\prime}\right), \\
& p_{2}=\sqrt{E} \operatorname{Im}\left(\lambda-\lambda^{\prime}\right)=\sqrt{E}\left(\sin \phi-\sin \phi^{\prime}\right),
\end{aligned}
$$

where $\lambda=e^{i \phi}, \lambda^{\prime}=e^{i \phi^{\prime}}$.

Proof of Proposition 4.2. Let $\lambda \in T, \lambda^{\prime} \in T$ be defined by (3.3). It follows from (3.5), (3.6) that the following formulas are valid:

$$
\begin{gathered}
2\left(k_{1}+l_{1}\right)=\sqrt{E}\left(\lambda+\lambda^{-1}+\lambda^{\prime}+\lambda^{\prime-1}\right), \\
2\left(k_{2}+l_{2}\right)=-i \sqrt{E}\left(\lambda-\lambda^{-1}+\lambda^{\prime}-\lambda^{\prime-1}\right), \\
k_{1} \pm i k_{2}=\sqrt{E} \lambda^{ \pm 1}, \quad l_{1} \pm i l_{2}=\sqrt{E} \lambda^{\prime \pm 1} \\
k_{1}+l_{2} \pm i\left(k_{2}+l_{2}\right)=\sqrt{E}\left(\lambda^{ \pm 1}+\lambda^{\prime \pm 1}\right) \\
|k+l|^{2}=E\left|\lambda+\lambda^{\prime}\right|^{2}
\end{gathered}
$$

where $(k, l) \in M_{E}$.

Using Lemma 7.1 and formulas (7.17) we derive from (2.21), (2.22), (2.23), 
(2.27), (2.28), (2.29) the following formulas:

$$
\begin{aligned}
A_{\text {appr }, \mathrm{j}}^{\mathrm{div}, 0}(x, E) & =\frac{E}{2} \int_{T^{2}} \widetilde{A}_{\mathrm{appr}, \mathrm{j}}^{\mathrm{div}, 0}\left(\lambda, \lambda^{\prime}, z, E\right)\left|\frac{1}{2 i}\left(\frac{\lambda}{\lambda^{\prime}}-\frac{\lambda^{\prime}}{\lambda}\right)\right||d \lambda|\left|d \lambda^{\prime}\right|, \\
V_{\mathrm{appr}}^{\mathrm{div}, 0}(x, E) & =\frac{E}{2} \int_{T^{2}} \widetilde{V}_{\mathrm{appr}}^{\mathrm{div}, 0}\left(\lambda, \lambda^{\prime}, z, E\right)\left|\frac{1}{2 i}\left(\frac{\lambda}{\lambda^{\prime}}-\frac{\lambda^{\prime}}{\lambda}\right)\right||d \lambda|\left|d \lambda^{\prime}\right|, \\
A_{\mathrm{appr}, 1}^{ \pm, 0}(x, E) & =\frac{E}{2} \int_{T^{2}} \widetilde{A}_{\mathrm{appr}, 1}^{ \pm, 0}\left(\lambda, \lambda^{\prime}, z, E\right)\left|\frac{1}{2 i}\left(\frac{\lambda}{\lambda^{\prime}}-\frac{\lambda^{\prime}}{\lambda}\right)\right||d \lambda|\left|d \lambda^{\prime}\right|, \\
V_{\mathrm{appr}}^{ \pm, 0}(x, E) & =\frac{E}{2} \int_{T^{2}} \widetilde{V}_{\mathrm{appr}}^{ \pm, 0}\left(\lambda, \lambda^{\prime}, z, E\right)\left|\frac{1}{2 i}\left(\frac{\lambda}{\lambda^{\prime}}-\frac{\lambda^{\prime}}{\lambda}\right)\right||d \lambda|\left|d \lambda^{\prime}\right|,
\end{aligned}
$$

where $j=1,2, x \in \mathbb{R}^{2}, 2, z$ is given by (3.2) and where

$$
\begin{aligned}
\widetilde{A}_{\text {appr }, 1}^{\text {div }, 0}\left(\lambda, \lambda^{\prime}, z, E\right) & =\frac{f^{\operatorname{lin}}\left(\lambda, \lambda^{\prime}, z, E\right)-f^{\operatorname{lin}}\left(-\lambda^{\prime},-\lambda, z, E\right)}{4 \sqrt{E}} \times \\
& \times\left(\frac{1}{\lambda^{-1}+\lambda^{\prime-1}}+\frac{1}{\lambda+\lambda^{\prime}}\right) \\
\widetilde{A}_{\text {appr }, 2}^{\text {div },}\left(\lambda, \lambda^{\prime}, z, E\right) & =\frac{f^{\operatorname{lin}}\left(\lambda, \lambda^{\prime}, z, E\right)-f^{\operatorname{lin}}\left(-\lambda^{\prime},-\lambda, z, E\right)}{4 i \sqrt{E}} \times \\
& \times\left(\frac{1}{\lambda^{-1}+\lambda^{\prime-1}}-\frac{1}{\lambda+\lambda^{\prime}}\right) \\
\widetilde{V}_{\text {appr }}^{\text {div, } 0}\left(\lambda, \lambda^{\prime}, z, E\right) & =\frac{f^{\operatorname{lin}}\left(\lambda, \lambda^{\prime}, z, E\right)+f^{\operatorname{lin}}\left(-\lambda^{\prime},-\lambda, z, E\right)}{2}, \\
\widetilde{A}_{\text {appr }, 1}^{ \pm, 0}\left(\lambda, \lambda^{\prime}, z, E\right) & =\frac{1}{2 \sqrt{E}} \frac{f\left(\lambda, \lambda^{\prime}, z, E\right)-f\left(-\lambda^{\prime},-\lambda, z, E\right)}{\lambda^{ \pm 1}+\lambda^{\prime \pm 1}}, \\
\widetilde{V}_{\text {appr }}^{ \pm, 0}\left(\lambda, \lambda^{\prime}, z, E\right) & =\frac{\lambda^{\prime \pm 1} f^{\operatorname{lin}}\left(\lambda, \lambda^{\prime}, z, E\right)+\lambda^{ \pm 1} f^{\operatorname{lin}}\left(-\lambda^{\prime},-\lambda, z, E\right)}{\lambda^{ \pm 1}+\lambda^{\prime \pm 1}},
\end{aligned}
$$

where $\lambda, \lambda^{\prime} \in T, z \in \mathbb{C}, f^{\operatorname{lin}}\left(\lambda, \lambda^{\prime}, z, E\right)$ is defined according to (4.7).

Now using (7.19) we represent each integral of (7.18) as a sum of the integral containing $f^{\operatorname{lin}}\left(\lambda, \lambda^{\prime}, z, E\right)$ and the integral containing $f^{\operatorname{lin}}\left(-\lambda^{\prime},-\lambda, z, E\right)$ within the integrands.

Making the change of variables $\left(\lambda, \lambda^{\prime}\right) \rightarrow\left(-\lambda^{\prime},-\lambda\right)$ in each integral contain$\operatorname{ing} f^{\text {lin }}\left(-\lambda^{\prime},-\lambda, z, E\right)$ and taking into account (2.27) for $\widehat{A_{2}^{ \pm}}$we obtain formulas (4.8), (4.9).

\section{References}

[ABF] M. J. Ablowitz, D. Bar Yaarov, A. S. Fokas, On the inverse scattering transform for the Kadomtsev-Petviashvili equation, Stud. Appl. Math. 69, 1983, 135-143 
[A] S. Arians, Geometric approach to inverse scattering for the Schrödinger equation with magnetic and electric potentials, J. Math. Phys. 38 (6), 1997, 2761-2773

[Ber] Yu. M. Berezansky, On uniqueness theorem in inverse problem of spectral analysis for Schrödinger equation, Mosc. Math. Soc. Proc. 7, 1958, 3-62 (in Russian)

[BBMRS] A. V. Bogatyrev, V. A. Burov, S. A. Morozov, O. D. Rumyantseva, E. G. Sukhov, Numerical realization of algorithm for exact solution of twodimensional monochromatic inverse problem of acoustical scattering, Acoustic Imaging 25, 2000, 65-70

[Buk] A.L. Bukhgeim, Recovering a potential from Cauchy data in the twodimensional case, J. Inverse Ill-Posed Probl. 16 (1), 2008, 19-33

[BBS] S. V. Baykov, V.A. Burov, S. N. Sergeev, Mode tomography of moving ocean, Proc. of the 3rd European Conference on Underwater Acoustics, $1996,845-850$.

[BAR] V. A. Burov, N. V. Alekseenko, O. D. Rumyantseva, Multifrequency generalization of the Novikov algorithm for the two-dimensional inverse scattering problem, Acoustical Physics 55 (6), 2009, 843-856

[ER1] G. Eskin, J. Ralston, Inverse scattering problem for the Schrödinger equation with magnetic potential at a fixed energy, Commun. Math. Phys. 173, 1995, 173-199

[ER2] G. Eskin, J. Ralston, Inverse scattering problems for Schrödinger operators with magnetic and electric potentials, Inverse problems in wave propagation, IMA Vol. Math. Appl. 90, 1997, 147-166

[DKN] B. A. Dubrovin, I. M. Krichever, S. P. Novikov, The Schrödinger equation in a periodic field and Riemann surfaces, Dokl. Akad. Nauk SSSR 229 (1), 1976, 15-18

[F1] L. D. Faddeev, Growing solutions of the Schrödinger equation, Dokl. Akad. Nauk SSSR 165 (3), 1965, 514-517

[F2] L. D. Faddeev, The inverse problem in the quantum theory of scattering. II, Journal of Mathematical Sciences 5 (3), 1976, 334-396

[GM] P.G. Grinevich, S. V. Manakov, The inverse scattering problem for the two-dimensional Schrödinger operator: The $\bar{\partial}$-method and non-linear equations, Funkt. Anal. i Pril. 20 (2), 1986, 14-24 (in Russian)

[GN1] P. G. Grinevich, R. G. Novikov, Analogues of multisoliton potentials for the two-dimensional Schrödinger operator, Funkt. Anal. i Pril. 19 (4), 1985, 32-42 (in Russian) 
[GN2] P. G. Grinevich, R. G. Novikov, Transparent potentials at fixed energy in dimension two. Fixed-energy dispersion relations for the fast decaying potentials, Commun. Math. Phys. 174 (2), 1995, 409-446

[GT] C. Guillarmou, L. Tzou, Identification of a connection from Cauchy data on a Riemann surface with boundary, Geom. Funct. Anal. 21 (2), 2011, $393-418$

[HN1] G. M. Henkin, R. G. Novikov, The $\bar{\partial}$-equation in the multidimensional inverse scattering problem, Uspekhi Mat. Nauk, 42 (3), 1987, 93-152 (in Russian); English Transl.: Russ. Math. Surv. 42 (3), 1987, 109-180

[HN2] G. M. Henkin, R. G. Novikov, A multidimensional inverse problem in quantum and acoustic scattering, Inverse Problems 8, 1988, 103-121

[IY] O. Yu. Imanuvilov, M. Yamamoto, Inverse problem by Cauchy data on an arbitrary sub-boundary for systems of elliptic equations, Inverse Problems 28, (2012), 095015 (30pp)

[M] S. V. Manakov, The inverse scattering transform for the time dependent Schrödinger equation and Kadomtsev-Petviashvili equation, Physica D 3 $(1,2), 1981,420-427$

[NSU] G. Nakamura, Z. Q. Sun, G. Uhlmann, Global identifiability for an inverse problem for the Schrödinger equation in a magnetic field, Math. Ann. 303 (3), (1995), 377-388

[Ni ] F. Nicoleau, A stationary approach to inverse scattering for Schrödinger operators with first order perturbation, Comm. Part. Diff. Eq. 22 (3-4), (1997), 527-553

[N1] R. G. Novikov, Construction of a two-dimensional Schrödinger operator with a given scattering amplitude at fixed energy, Teoret. Mat. Fiz. 66, 1986, 234-240 (in Russian)

[N2] R. G. Novikov, Multidimensional inverse spectral problem for the equation $-\Delta \psi+(v(x)-E u(x)) \psi=0$, Funkt. Anal. i Pril. 22 (4), 1988, 11-22 (in Russian); English Transl.: Funct. Anal. and Appl. 22, 1988, 263-272

[N3] R. G. Novikov, The inverse scattering problem on a fixed energy level for the two-dimensional Schrödinger operator, J. Funct. Anal. 103 (2), 1992, 409-469

[N4] R. G. Novikov, Approximate solution of the inverse problem of quantum scattering theory with fixed energy in dimension 2, Proceedings of the Steklov Institute of Mathematics, 225 (2), 1999, 285-302

[NS] R. G. Novikov, M. Santacesaria, Monochromatic reconstruction algorithms for two-dimensional multi-channel inverse problems, Int. Math. Res. Not. IMRN 6, 2013, 1205-1229 
[Nor] S. J. Norton, Reconstruction of stratified fluid flow from reciprocal scattering measurements, J. Acoust. Soc. Am. 89 (6), 1991, 2567-2572

[RW] D. Roussef, K. B. Winters, Two-dimensional vector flow inversion by diffraction tomography, Inverse Problems 10, 1994, 687-697

[RE] M. N. Rychagov, H. Ermert, Reconstruction of fluid motion in acoustic diffraction tomography, J. Acoust. Soc. Am. 99 (5), 1996, 3029-3035

[Sh] T. Shiota, An inverse problem for the wave equation with first order perturbation, Amer. J. Math. 107 (1), 1985, 241-251

[WY] R. Weder, D. Yafaev, On inverse scattering at a fixed energy for potentials with a regular behaviour at infinity, Inverse Problems 21, 2005, 1937-1952

[X] Xiaosheng Li, Inverse scattering problem for the Schrödinger operator with external Yang-Mills potentials in two dimensions at fixed energy, Comm. Part. Diff. Eq. 30, 2005, 451-482 